\title{
Modelling the influence of strain localisation and viscosity on the behaviour of underground drifts drilled in claystone
}

\author{
B. Pardoen ${ }^{\mathrm{a}, 1}$, F. Collin ${ }^{\mathrm{a}, *}$ \\ ${ }^{a}$ ArGEnCo Department, University of Liège, Allée de la Découverte 9, 4000 Liège, Belgium
}

\begin{abstract}
In the context of the deep geological repository of nuclear wastes, Andra conducts a benchmark called "Transverse action" to characterise and model the behaviour of the Callovo-Oxfordian claystone. The main objectives are to develop and calibrate numerical models with the purpose of modelling underground structures, by the reproduction of the drilling phase and Excavation Damaged Zone (EDZ) around the galleries. The proposed model is a crossanisotropic elasto-viscoplastic model including the anisotropy of both elastic and plastic behaviours. A viscoplastic mechanism is considered to reproduce the creep deformation of the material in the long term. The parameters of the model are calibrated based on laboratory test results provided by Andra. Once the model is calibrated, the drilling process at large scale is reproduced with a finite element method. The shear fractures that develop around the galleries are reproduced with strain localisation in shear bands. The purpose of the modelling is to highlight the contributions of shear banding, viscosity, and material anisotropy for the reproduction of the EDZ and claystone behaviour at repository scale. The numerical modelling indicates that the EDZ and the gallery convergence can not be correctly represented if the fractures are not reproduced. A classical continuous approach without shear band modelling fails
\end{abstract}

\footnotetext{
*Corresponding author. Tel.: +32 43669142.

Email address: f.collin@ulg.ac.be (F. Collin)

${ }^{1}$ FRIA, F.R.S.-FNRS scholarship holder
} 
to reproduce the EDZ behaviour. Modelling shear banding around the gallery is an important aspect that leads to a better description of the EDZ for the considered claystone. Moreover, considering the material viscosity allows a better reproduction of the gallery convergence in the long term.

Keywords: Excavation damaged zone, strain localisation, shear banding, viscosity, cross-anisotropy, numerical modelling

\section{Introduction}

The underground repository of nuclear wastes in deep geological formations is nowadays a crucial issues. To assure a safe repository on the long term, the behaviour of the possible host formations and of the underground structures, 5 where the most hazardous type of wastes will be stored, need to be defined and predicted. The underground structures are composed of a network of galleries whose drilling inevitably engenders stress redistribution, damage, and fractures in the surrounding medium. Different low-permeability host materials are envisaged for the deep geological repository and, among them, the behaviour and drilling of the Callovo-Oxfordian claystone is studied. This geological medium is studied in France by the national radioactive waste management agency Andra. Andra conducts various scientific research programs to ensure the feasibility of a safe repository in the Callovo-Oxfordian claystone. The benchmark "Transverse action" consists in developing numerical models to characterise the claystone behaviour, to calibrate them based on experimental results, and finally to use them to numerically model underground structures by the reproduction of the drilling phase.

Many experimental measurements and observations in the considered argillaceous rock have highlighted that damage and fracturing around the galleries play an important role in the hydro-mechanical behaviour of the Excavation Damaged Zone (EDZ) 1, 2]. The latter is a zone that develops around underground galleries due to the drilling process and which is dominated by irreversible hydromechanical property changes. The shape and extent of the EDZ is also domi- 
nated by the material anisotropy either of the in situ initial stress state or of the mechanical behaviour. Taking into account the material anisotropy and the representation of the fractures are therefore necessary to correctly predict the behaviour of the EDZ.

The proposed constitutive model is a cross-anisotropic and elasto-viscoplastic model. The anisotropy is considered both for the elastic and plastic behaviours of the material. For the plastic behaviour, the evolution of a strength parameter is considered with a microstructure fabric tensor. Creep deformations are also considered by the introduction of a viscoplastic mechanism in order to reproduce the increase of gallery convergence which is observed in the long term. The different aspects of the model are calibrated based on experimental results 35 obtained in laboratory, on small-scale samples, and provided by Andra.

Among various possibilities to represent the fractures, it is chosen to represent them at large scale around galleries with strain localisation in shear bands [3. This approach is chosen because shear banding is usually observed in geomaterials and develops before localised rupture or fractures [4]. Furthermore, the fracturing process in the Callovo-Oxfordian claystone is dominated by shear fractures around the galleries [2]; thus, shear banding can be an adequate manner to represent these fractures [5].

The proposed approach aims to highlight the contributions of material anisotropy, viscosity, and shear banding in the reproduction of the claystone behaviour as 45 well as in the development of the EDZ. The numerical modelling are realised with the non-linear finite element code Lagamine developed at the University of Liège [6]. The results indicate that the EDZ behaviour and the gallery convergence can not be well represented without considering the reproduction of the fractures in the rock.

\section{so 2. Constitutive models}

The Callovo-Oxfordian claystone is assumed to be a biphasic porous medium composed of solid particles and liquid water under unsaturated conditions. The 
constitutive equations of the hydro-mechanical model are described in this section. Firstly, the balance equations are established, then the mechanical and

55 hydraulic models are detailed for a cross-anisotropic rock exhibiting creep deformation. Moreover, gravity is not taken into account in the problem.

\subsection{Balance equations}

The balance equations are described hereafter for classical poromechanics defined at macroscale and for microstructure medium. The latter is relevant for the modelling of strain localisation in geomaterials.

\subsubsection{Classical medium}

The balance equations of the mixture momentum and of the water mass are:

$$
\begin{gathered}
\int_{\Omega} \sigma_{i j} \epsilon_{i j}^{*} d \Omega=\int_{\Gamma_{\sigma}} \bar{t}_{i} u_{i}^{*} d \Gamma \\
\int_{\Omega}\left(\dot{M}_{w} p_{w}^{*}-f_{w, i} \frac{\partial p_{w}^{*}}{\partial x_{i}}\right) d \Omega=\int_{\Omega} Q_{w} p_{w}^{*} d \Omega-\int_{\Gamma_{q_{w}}} \bar{q}_{w} p_{w}^{*} d \Gamma
\end{gathered}
$$

where the general notation $a^{*}$ represents the kinematically admissible virtual quantity $a, \Omega$ is the porous material configuration, $u_{i}$ is the macroscale displacement field, $\epsilon_{i j}$ is the strain field, $\sigma_{i j}$ is the total stress field $\left(\sigma_{i j}>0\right.$ for

${ }_{65}$ compression), and $\bar{t}_{i}$ is the classical external traction force per unit area acting on a part $\Gamma_{\sigma}$ of the total boundary $\Gamma$. Further, $p_{w}$ is the pore water pressure field ( $p_{w}<0$ if suction), $f_{w, i}$ is the mass flow of water, $\dot{M}_{w}$ is the variation of the water mass, $\bar{q}_{w}$ is an input water mass per unit area on a part $\Gamma_{q_{w}}$ of $\Gamma$, and $Q_{w}$ is a water sink term.

The effective stress field $\sigma_{i j}^{\prime}$ is defined by Biot's postulate [7] under unsaturated conditions and takes into account the solid phase compressibility:

$$
\sigma_{i j}=\sigma_{i j}^{\prime}+b_{i j} S_{r, w} p_{w}
$$
degree of water saturation. 


\subsubsection{Microstructure enhanced medium}

In geomaterials, shear strain localisation is often observed before the localised failure of the material and fracturing. When subjected to external solicitations, homogeneous deformation are firstly visible in geomaterials; then, once the damage threshold is reached, material damage by microcracking develops. Further, if microcracks coalesce, it can lead to strain localisation in shear bands and to the onset of interconnected fractures (macrocracks). It is therefore proposed to represent the shear fractures around the galleries in a continuous manner (weak discontinuities) with strain localisation in shear bands [8, 9].

In classical finite element methods, the strain localisation suffers a mesh sensitivity [10] which can be avoided by regularising the numerical method with the introduction of an internal length scale. The latter allows a proper reproduction of the post-localisation material behaviour. Among the various regularisation 85 methods that exist, an enrichment with microstructure kinematics is considered [11, 12. The coupled local second gradient model is used and introduces microscale kinematics in addition to the classical macroscale kinematics [13, 14, 3].

The momentum balance equation of the mixture is:

$$
\int_{\Omega}\left(\sigma_{i j} \frac{\partial u_{i}^{*}}{\partial x_{j}}+\Sigma_{i j k} \frac{\partial v_{i j}^{*}}{\partial x_{k}}\right) d \Omega=\int_{\Gamma_{\sigma}}\left(\bar{t}_{i} u_{i}^{*}+\bar{T}_{i} v_{i k}^{*} n_{k}\right) d \Gamma
$$

where $\Sigma_{i j k}$ is the double stress, $v_{i j}=\frac{\partial u_{i}}{\partial x_{j}}$ is the gradient field of the microkinematics, $n_{k}$ is the normal unit vector to the boundary, and $\bar{T}_{i}$ is the additional external double force per unit area. The double stress $\Sigma_{i j k}$ involves an additional constitutive law in which the internal length scale is introduced.

It is assumed that the pore fluid does not have an influence at microscale [15. Second gradient effects are only assumed for the solid phase and the water mass balance equation 2 of classical poromechanics is conserved.

95 2.2. Mechanical models

The Callovo-Oxfordian claystone is a sedimentary material which exhibits a cross-anisotropy, i.e. transverse isotropy, with quasi-horizontal isotropic planes 
(bedding planes). Creep deformations have also been observed for this material. Therefore, the rock behaviour is defined with an elasto-viscoplastic hydromechanical model including cross-anisotropy.

\subsubsection{First gradient}

The elasto-plastic behaviour is defined for a transversely isotropic material, which properties depend on the loading orientation relative to the preferential axes of the material structure.

The elastic law is classically defined with the Hooke's linear relation [16, 17. and the compressibility of the solid grains is defined through the Biot's tensor [18. The parameters that describe the elasticity are detailed in Table 2 for the considered rock. The subscripts $\|$ and $\perp$ indicate the directions parallel and perpendicular to the isotropic planes, respectively.

A non-associated plasticity is considered with a plastic behaviour defined by an internal friction model and by a Van Eekelen yield surface [19]:

$$
F \equiv I I_{\hat{\sigma}}-m\left(I_{\sigma^{\prime}}+\frac{3 c}{\tan \varphi_{c}}\right)=0
$$

where $c$ is the rock cohesion, $\varphi_{c}$ is the friction angle in compression, $I_{\sigma^{\prime}}$ and $I I_{\hat{\sigma}}$ are the first and second stress invariants, and $m$ is a model parameter which introduces the dependence to the Lode angle. An isotropic hardening or softening is allowed for the cohesion and the friction angles. The evolutions of the strength parameters follow hyperbolic functions [20] which are defined as follows:

$$
a=a_{0}+\frac{\left(a_{f}-a_{0}\right)\left\langle\hat{\epsilon}_{e q}^{p}-d e c_{a}\right\rangle}{B_{a}+\left\langle\hat{\epsilon}_{e q}^{p}-d e c_{a}\right\rangle}
$$

110

$\hat{\epsilon}_{e q}^{p}=\sqrt{\frac{2}{3} \hat{\epsilon}_{i j}^{p} \hat{\epsilon}_{i j}^{p}}$ is the Von Mises' equivalent deviatoric plastic strain, $\hat{\epsilon}_{i j}^{p}=$ $\epsilon_{i j}^{p}-\frac{\epsilon_{k k}^{p}}{3} \delta_{i j}$ is the deviatoric part of the plastic strain tensor $\epsilon_{i j}^{p}, B_{a}$ is an evolution parameter, and $d e c_{a}$ is the value of $\hat{\epsilon}_{e q}^{p}$ from which hardening/softening starts.

The inherent anisotropy of the plastic behaviour is introduced with a second order fabric tensor $A_{i j}$ which represents the microstructure of the material 
[21, 22, 23]. The anisotropy and spacial distribution of the cohesion are defined with this tensor. Moreover, the cohesion also depends on the direction of loading relative to the orientation of the material microstructure [24]. For cross-anisotropic rocks, the evolution of the initial and final cohesions, $c_{0}$ and $c_{f}$, are given by:

$$
\begin{gathered}
c_{0}=\bar{c}\left(1+A_{\|\|}\left(1-3 l_{\|}^{2}\right)+b_{1} A_{\|\|}^{2}\left(1-3 l_{\|}^{2}\right)^{2}\right) \\
c_{f}=\frac{c_{0}}{\xi}
\end{gathered}
$$

115 where $A_{\|\mid\|}$is the component of the microstructure fabric tensor in the isotropic planes, $\bar{c}$ and $b_{1}$ are constant parameters, $\xi$ is the ratio of cohesion softening, and $l_{\|}$is the component of a generalised unit loading vector $l_{i}[25]$ on a facet parallel to the isotropic planes. The loading vector is defined as the resultant of the stress state on the material facets.

Viscoplasticity is also taken into account to reproduce the creep deformation of the claystone [26, 27. The plastic strain is assumed to be composed of an instantaneous strain $\epsilon_{i j}^{p}$ independent of time and of a time-dependent creep strain $\epsilon_{i j}^{v p}$ [28]. The viscoplastic loading surface is defined as [29, 30]:

$$
F^{v p} \equiv \sqrt{3} I I_{\hat{\sigma}}-\alpha^{v p} R_{c} \sqrt{A\left(C_{s}+\frac{I_{\sigma^{\prime}}}{3 R_{c}}\right)}=0
$$

where $R_{c}$ is the uniaxial compressive strength, $A$ is an internal friction coefficient, $C_{s}$ is a constant cohesion coefficient, $\alpha^{v p}$ is a delayed hardening function defined as:

$$
\alpha^{v p}=\alpha_{0}^{v p}+\left(1-\alpha_{0}^{v p}\right) \frac{\epsilon_{e q}^{v p}}{B^{v p}+\epsilon_{e q}^{v p}}
$$

120

where $\alpha_{0}^{v p}$ is the initial threshold for the viscoplastic flow, $B^{v p}$ is a hardening parameter, and $\epsilon_{e q}^{v p}$ is the equivalent viscoplastic strain. A complete description of the viscoplastic model is available in 29 and 30 .

\subsubsection{Second gradient}

For microstructure media, a constitutive stress-strain relation between the kinematics and the stress at microscale is required in addition to the classical 
stress-strain relation. This law relates the rate of the micro second gradient $\frac{\partial \dot{v}_{i j}}{\partial x_{k}}$ to the Jaumann double stress rate $\tilde{\Sigma}_{i j k}$. It is an isotropic linear elastic equation defined in [31]:

$$
\tilde{\Sigma}_{i j k}=D_{i j k l m n} \frac{\partial \dot{v}_{l m}}{\partial x_{n}}
$$

This second gradient law is decoupled of the classical first gradient part and it is assumed that the pore fluid does not have an influence at the microscale. Thus, the second gradient mechanical law is not related to the pore water pressure. The matrix $D_{i j k l m n}$ in Eq. 11 is composed of only one elastic parameter $D$ in its simplified version proposed in [32]. This elastic modulus represents the physical microstructure of the material and is linked to an internal length scale introduced to regularise the post-localisation material behaviour [33, 34]. The shear band width is related to this parameter which allows a correct representation of the strain localisation process by restoring mesh objectivity [14].

\subsection{Hydraulic model}

The transfer of the liquid water by advection is modelled in unsaturated porous media by the generalised Darcy's law. The latter depends on the anisotropic tensor of saturated (intrinsic) water permeability $k_{i j}$ and on the relative water permeability $k_{r, w}$. For cross-anisotropic rocks, $k_{i j}$ is defined by $k_{\|\|}$and $k_{\perp \perp}$. Furthermore, the partial saturation of the rock is defined by a relative water permeability curve and a retention curve from Mualem - van Genuchten's model 140 35.

\section{Calibration of model parameters}

The Callovo-Oxfordian claystone is a sedimentary material having horizontal bedding planes. Thus, the main orientations of anisotropy are located in the horizontal isotropic planes and along the vertical direction. It goes without saying that the calibration of the claystone parameters has to take into account these preferential material directions. 
The anisotropic and elasto-viscoplastic mechanical parameters are calibrated based on compression tests performed in laboratory on small-scale samples. For these tests, the global stress-strain curves of the specimen response exhibit peak stresses that corresponds to the appearance of shear bands in the samples. After the peak stress, the post-peak behaviour is governed mainly by the development of shear bands and shear fractures within the samples.

The reproduction of shear bands in the material should be considered to calibrate the model parameters. The calibration would depend upon the structure of shear bands in the Callovo-Oxfordian claystone. Unfortunately, complete experimental information on shear banding is seldom available for rocks. Furthermore, the strain localisation process is a three-dimensional problem that would require three-dimensional numerical modelling to accurately predict the post-peak behaviour. The latter is quite complex and requires to extend the second gradient model to three-dimensional modelling which is beyond the scope of the present study.

A more classical approach is to consider the material as homogeneous. For this approach, the peak stress and post-peak behaviour can be represented by the softening of strength parameters in the constitutive model. Due to the complexity of relating the model calibration to shear banding, the homogeneous approach is considered for classical material (section 2.1.1). Localised solutions with shear bands will be analysed in the numerical modelling of the gallery excavation problem in section 4.3 .

The calibration of the model parameters is envisaged in different steps. The hydraulic parameters and the elastic mechanical parameters are described at first, taking into account their anisotropy. Then, the plastic mechanical parameters are evaluated based on experimental results (section 3.3). Firstly, the hardening and softening of the plastic behaviour under compression is analysed with anisotropic elasticity and isotropic plasticity (no cohesion anisotropy). This first calibration considers that the post-peak behaviour is reproduced with cohesion softening for a homogeneous material. As mentioned previously, the post-peak behaviour should be related to the appearance of shear banding. Moreover, a 
Table 1: Hydraulic parameters.

\begin{tabular}{llll}
\hline Symbol & Name & Value & Unit \\
\hline$k_{\|\|}$ & Parallel intrinsic water permeability & $4 \times 10^{-20}$ & $\mathrm{~m}^{2}$ \\
$k_{\perp \perp}$ & Perpendicular intrinsic water permeability & $1.33 \times 10^{-20}$ & $\mathrm{~m}^{2}$ \\
$\Phi$ & Porosity & 0.173 & - \\
$P_{r}$ & Air entry pressure & 15 & $\mathrm{MPa}$ \\
$\mathcal{M}$ & van Genuchten coefficient & 0.33 & - \\
$S_{m a x}$ & Maximum degree of water saturation & 1 & - \\
$S_{r e s}$ & Residual degree of water saturation & 0.01 & - \\
$\chi_{w}^{-1}$ & Water compressibility & $5 \times 10^{-10}$ & $\mathrm{~Pa}^{-1}$ \\
\hline
\end{tabular}

softening behaviour engenders the appropriate conditions for the onset of strain localisation. Consequently, the softening parameters have to be adapted to trigger strain localisation for the considered application of a gallery excavation. The cohesion softening will therefore not be reproduced for the other calibrations at small scale and shear banding will be modelled later for the gallery drilling. Secondly, after the calibration of the plastic properties under compression, the extensional plastic properties are evaluated. Thirdly, the parameters of the cohesion anisotropy are calibrated. Finally, the viscoplastic parameters are evaluated in section 3.4 .

\subsection{Hydraulic parameters}

The hydraulic parameters of the Callovo-Oxfordian claystone required for the model come from [36] where a synthesis of the claystone parameters is detailed. The calibration is obtained from laboratory experiments and from results available in the literature. The parameters are summarised in Table 1

\subsection{Elastic parameters}

To evaluate the anisotropic elastic parameters, compression experiments have been realised on samples for different loading directions with regard to 
Table 2: Anisotropic elastic mechanical parameters.

\begin{tabular}{llll}
\hline Symbol & Name & Value & Unit \\
\hline$E_{\|}$ & Parallel Young's modulus & 5 & $G P a$ \\
$E_{\perp}$ & Perpendicular Young's modulus & 4 & $G P a$ \\
$G_{\| \perp}$ & Shear modulus & 1.92 & $G P a$ \\
$\nu_{\|\|}$ & Poisson's ratio & 0.3 & - \\
$\nu_{\perp \|}$ & Poisson's ratio & 0.24 & - \\
$b_{\|\|}$ & Parallel Biot's coefficient & 0.60 & - \\
$b_{\perp \perp}$ & Perpendicular Biot's coefficient & 0.66 & - \\
$\rho_{s}$ & Solid grain density & 2750 & $\mathrm{~kg} / \mathrm{m}^{3}$ \\
\hline
\end{tabular}

195

$b_{\perp \perp}=0.66$ by the micro-isotropy assumed in Eq. ??.

\subsection{Plastic parameters}

The calibration of the plastic parameters is realised based on the experimental data provided by Andra in the context of the benchmark and on additional compression tests. The different experimental results are detailed in this section together with the calibration of the numerical model. 


\subsubsection{Hardening and softening}

The hardening and softening plastic behaviour of the Callovo-Oxfordian claystone is calibrated based on triaxial compression tests. The reference tests are performed at different confining pressures, under a relative humidity of $R H=90 \%$, and are assumed to be realised under undrained conditions. Their characteristics and results are available in Table 3 and Fig. 1, where $\epsilon_{1}$ is the axial strain, $\epsilon_{3}$ is the lateral strain, and $\underline{q}$ is the deviatoric stress corresponding to the difference between the axial stress $\sigma_{1}$ and the confining stress $\sigma_{3}$ :

$$
\underline{q}=\sigma_{1}-\sigma_{3}
$$

The tests are numerically reproduced by finite element method, with a hydro-mechanical modelling in two-dimensional axisymetric state. The sam-14.2 MPa by Kelvin's law (by taking into account only the capillary effects) and to $S_{r, w}=81 \%$ by the retention curve of the material. Kelvin's law assumes an instantaneous hydraulic equilibrium between liquid water and water vapour inside a porous material. Furthermore, the material is considered with an anisotropic elasticity and an isotropic plasticity (no cohesion anisotropy) to calibrate firstly the hardening/softening parameters.

On the experimental curves of Fig. 1, one can observe that the pre-peak behaviour is not linear which can be related to the damage of elastic properties or to the hardening of plastic properties. Damage of material characteristics is not taken into account in the model; then, the pre-peak behaviour can be captured by hardening of a strength parameter. The friction angle and the cohesion define the plastic criterion and the friction angle is chosen as the hardening variable. The post-peak behaviour highlights a material strength decrease that will be captured by softening of a plastic characteristic. For granular materials, it can be assumed that the friction angle does not decrease significantly in the postpeak regime. On the other hand, the cohesion is affected and a lower residual value is generally observed. As a consequence, the residual strength is mostly affected by the cohesion which is therefore chosen as the softening variable, and 
Table 3: Triaxial compression tests and calibration of plastic parameters for elastoplastic mechanical model.

\begin{tabular}{|c|c|c|c|c|c|c|c|c|}
\hline Symbol & Name & Triax 01 & Triax 02 & Triax 03 & Triax 04 & Triax 05 & Average & Unit \\
\hline & Laboratory & LML & LML & LML & LAEGO & LAEGO & & \\
\hline$R H$ & Relative humidity & 90 & 90 & 90 & & & & $\%$ \\
\hline$\dot{\epsilon}$ & Strain rate & $1 \times 10^{-6}$ & $1 \times 10^{-6}$ & $1 \times 10^{-6}$ & $20 \times 10^{-6}$ & $3.5 \times 10^{-6}$ & & $s^{-1}$ \\
\hline$\sigma_{3}$ & Confining pressure & 12 & 12 & 6 & 2 & 12 & & $M P a$ \\
\hline$\alpha$ & $\begin{array}{l}\text { Orientation of } \\
\text { the loading }\end{array}$ & $\perp$ & $\|$ & $\perp$ & $\perp$ & $\perp$ & & $\circ$ \\
\hline$\psi_{c}$ & $\begin{array}{l}\text { Dilatancy angle } \\
\text { in compression }\end{array}$ & 0.5 & 0.5 & 0.5 & 0.5 & 0.5 & 0.5 & $\circ$ \\
\hline$\varphi_{c, 0}$ & $\begin{array}{l}\text { Initial compression } \\
\text { friction angle }\end{array}$ & 10 & 10 & 10 & 10 & 6 & 10 & $\circ$ \\
\hline$\varphi_{c, f}$ & $\begin{array}{l}\text { Final compression } \\
\text { friction angle }\end{array}$ & 23 & 22 & 24 & 23 & 24 & 23 & $\circ$ \\
\hline$B_{\varphi}$ & $\begin{array}{l}\text { Friction angle } \\
\text { hardening coefficient }\end{array}$ & 0.0010 & 0.0015 & 0.0007 & 0.0005 & 0.0015 & 0.0010 & - \\
\hline $\operatorname{dec}_{\varphi}$ & $\begin{array}{l}\text { Friction angle } \\
\text { hardening shifting }\end{array}$ & 0 & 0 & 0 & 0 & 0 & 0 & - \\
\hline$c_{0}$ & Initial cohesion & 4 & 4 & 4 & 4.5 & 5 & 4.2 & $M P a$ \\
\hline$c_{f}$ & Final cohesion & 1 & 0.04 & 0.04 & 1 & 2.5 & $0.04-2$ & $M P a$ \\
\hline$B_{c}$ & $\begin{array}{l}\text { Cohesion softening } \\
\text { coefficient }\end{array}$ & 0.001 & 0.0005 & 0.002 & 0.002 & 0.001 & 0.001 & - \\
\hline$d e c_{c}$ & $\begin{array}{l}\text { Cohesion softening } \\
\text { shifting }\end{array}$ & 0.016 & 0.014 & 0.007 & 0.004 & 0.015 & 0.011 & - \\
\hline
\end{tabular}



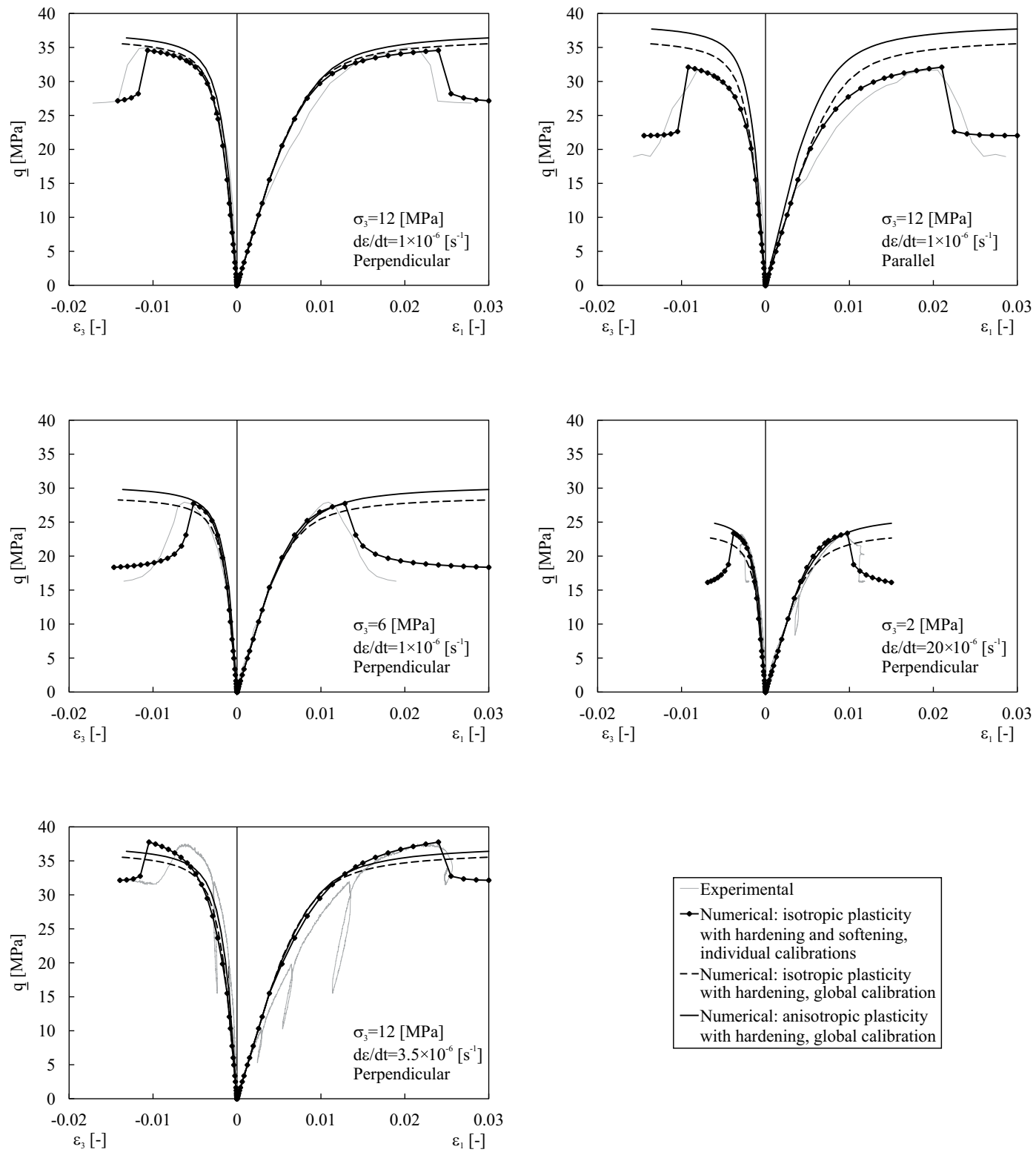

\begin{tabular}{|l|}
\hline Experimental \\
$\rightarrow$-Numerical: isotropic plasticity \\
with hardening and softening, \\
individual calibrations \\
- -Numerical: isotropic plasticity \\
with hardening, global calibration \\
- Numerical: anisotropic plasticity \\
with hardening, global calibration
\end{tabular}

Figure 1: Fitting of triaxial compression tests for elastoplastic mechanical model. 
no softening of the friction angle is assumed. The pre-peak non-linear behaviour is therefore controlled by friction angle hardening and the post-peak behaviour is represented by cohesion softening. The start of the softening can be delayed to reproduce the position of the peak stress on the global response curve of the specimens. This is realised thanks to the softening shifting parameter for the cohesion, $\operatorname{dec}_{c}$ (Eq. 6).

Because the material is subjected to compression, only the compressive resistance is calibrated. The calibration results for each individual triaxial compression test are available in Fig. 1 1 and Table 3 where one can observe that the values of $c_{f}$ are widely scattered. However, as mentioned previously, cohesion softening may results in the appearance of strain localisation and non-homogeneous solutions. Therefore, it will be introduced later to model shear banding in rock (section 4.3). Only the friction angle hardening is included in the following calibrations for a homogeneous material. The average values of Table 3 are kept for the hardening parameters and a global calibration with this set of parameters is illustrated in Fig. 1, without softening. In that figure are also detailed the results for an anisotropic plasticity which will be discussed further.

\subsubsection{Extensional properties}

The extensional material properties are evaluated from triaxial extension tests that are performed at constant mean stress $\sigma=\frac{\sigma_{i i}}{3}$. Two test results are analysed, one with $\sigma=12 \mathrm{MPa}$ and another with $\sigma=13 \mathrm{MPa}$, for an axial load perpendicular to the bedding planes and assuming a relative humidity of $R H=90 \%$ as well as undrained conditions (as for the triaxial compression tests). During these tests, the axial load decreases and the confining pressure increases; therefore, the extension is in the axial direction.

The modelling is performed for an isotropic plasticity and with the average parameters of Table 3 The numerical results without cohesion softening are illustrated in Fig. 2 where they are compared to the experimental results. The final friction angles in compression and extension are equal but the initial friction angle in extension is decreased to better match the experimental curves: $\varphi_{e, f}=$ 

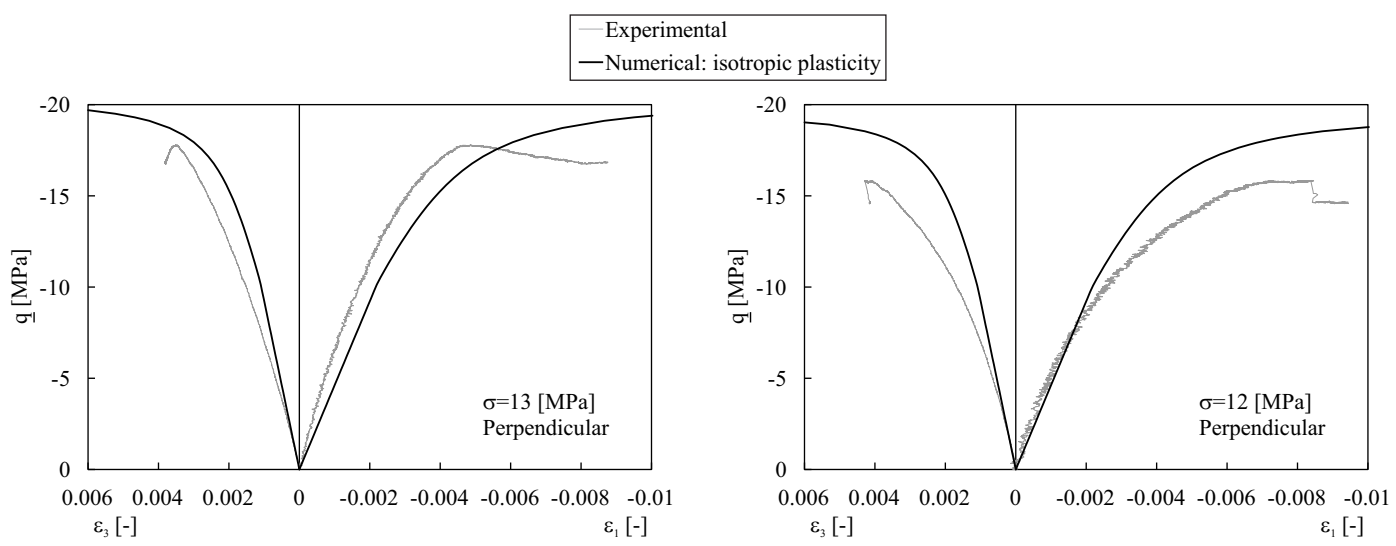

Figure 2: Fitting of triaxial extension tests for elastoplastic mechanical model.

$\varphi_{c, f}=23^{\circ}, \varphi_{e, 0}=7^{\circ}$, and $\varphi_{c, 0}=10^{\circ}$. The other hardening parameters $B_{\varphi}$ and $\operatorname{dec}_{\varphi}$ are the same in extension than in compression (Table 3). The dilatancy angles in compression and extension are also equal $\psi_{c}=\psi_{e}=0.5^{\circ}$.

\subsubsection{Anisotropy of cohesion}

Among the triaxial compression tests provided by Andra, only one is performed with a compression in the direction parallel to the isotropic planes. Consequently, the calibration of the anisotropic plastic parameters for the cohesion in Eq. 7 requires additional compression experiments. Based on uniaxial compression results, an evolution of the cohesion of the Callovo-Oxfordian claystone with the loading orientation is proposed in [5]. The cohesion evolves according to Fig. 3 where $\alpha$ corresponds to the angle between the compression direction and the normal to the bedding planes. It can be observed that the minimum value of the cohesion occurs for a loading of about $45^{\circ}$ which corresponds to experimental results on other transversal isotropic argillaceous rock. For this evolution, the constants of the microstructure fabric tensor (Eq. 7) are: $\bar{c}=4.1 \mathrm{MPa}, A_{\|\| \|}=0.117$, and $b_{1}=14.24$.

280 To validate these anisotropic parameters, the triaxial compression tests used to determine the hardening and softening parameters can be modelled with the anisotropic model. Only one global fitting is performed with the Van Eeke- 


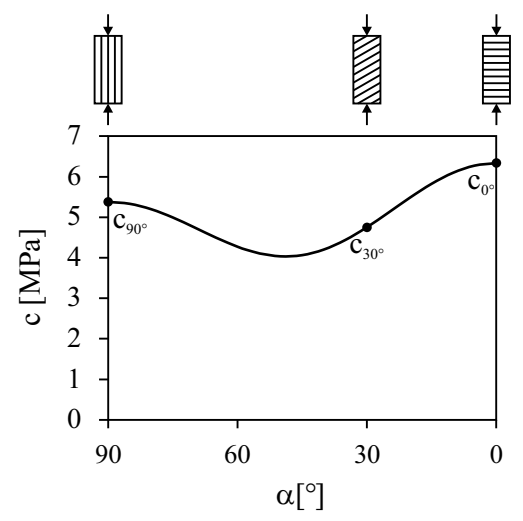

Figure 3: Evolution of the material cohesion with the angle between the direction of loading and the normal to the isotropic bedding planes [5].

len criterion and without softening; the results are available in Fig. 1. Among the five triaxial compression tests, the resistance is overestimated only for the compression test performed parallel to the bedding planes. To improve this, the value of the cohesion parallel to the bedding planes $c_{90^{\circ}}$ can be decreased without altering the reproduction of the compression tests performed perpendicularly to the bedding. Nevertheless, the reduction of the cohesion $c_{90^{\circ}}$ leads to a cohesion evolution that does not correspond to classical measured values for the considered material because it does not exhibit a lower value around $\alpha=45^{\circ}$ anymore. Besides, only one compression test performed parallel to the stratification is modelled here which does not capture the dispersion of laboratory results. On the other hand, the anisotropic cohesion parameters determined from the uniaxial compression tests come from a large number of laboratory tests. Consequently, the first set of parameters seems to better suit for the material and is kept.

\subsection{Viscoplastic parameters}

As for the plastic parameters, the viscoplastic parameters are determined based on experimental data provided by Andra in the context of the benchmark. Additionally, typical values of viscoplastic parameters for the Callovo-Oxfordian claystone under $-450 \mathrm{~m}$ depth are available in [29]. Those parameters come 
from a particular fitting procedure and are therefore adjusted based on the provided reference creep tests. These creep tests are performed at a confining pressure of $12 \mathrm{MPa}$ and at different constant stress deviators to highlight creep deformation in the long term. The stress deviators $\underline{q}=\sigma_{1}-\sigma_{3}$ correspond to $50 \%, 75 \%$, and $90 \%$ of a considered deviatoric peak stress of $34 \mathrm{MPa}$, which gives $17,25.5$, and $30.6 \mathrm{MPa}$. Once again, a relative humidity of $90 \%$ is considered however drained conditions are assumed due to the long-term testing procedure. To reproduce the constant stresses in the different directions, the tests are numerically modelled at small scale with a three-dimensional structure corresponding to one cubic finite element. A hydro-mechanical modelling is realised, with the average values of Table 3 for the friction angle hardening parameters and no cohesion softening.

To calibrate the different parameters, the value of the initial threshold for the creep deformation $\alpha_{0}^{v p}$ in Eq. 10 has to be identified firstly. It should be determined at the onset of irreversible creep strains during compression tests but, in practice, it is not simple to define such point precisely. It is therefore assumed that creep strains start at low deviatoric stress for hard clay [30] and two types of fitting are analysed. First, in agreement with instantaneous viscoplastic deformation mechanism, a value of $\alpha_{0}^{v p}=0$ can be chosen and leads to an initial viscoplastic flow $F_{0}^{v p}=\sqrt{3} I I_{\hat{\sigma}, 0}$ by Eq. 9. The initial second deviatoric stress invariant $I I_{\hat{\sigma}, 0}$ and initial viscoplastic flow $F_{0}^{v p}$ cancel only in case of initial isotropic effective stress state. Second, anticipating the further modelling of a gallery excavation in the Callovo-Oxfordian claystone, its initial anisotropic stresses and pore water pressure are considered. In order to avoid an initial viscoplastic flow in the rock mass, $F_{0}^{v p}=0$, the value of $\alpha_{0}^{v p}$ is chosen as (Eq. 9):

$$
\alpha_{0}^{v p}=\frac{\sqrt{3} I I_{\hat{\sigma}, 0}}{R_{c} \sqrt{A\left(C_{s}+\frac{I_{\sigma^{\prime}, 0}}{3 R_{c}}\right)}}
$$

where the values of the parameters come from [29]: $R_{c}=21 \mathrm{MPa}, A=2.62$, $C_{s}=0.03 ;$ and the initial stress invariants, $I_{\sigma^{\prime}, 0}$ and $I I_{\hat{\sigma}, 0}$, are determined from 
the initial in situ effective stress state of the argillaceous rock. In the context of the Andra's benchmark, the recommended anisotropic total stress state and pore water pressure are:

$$
\begin{gathered}
\sigma_{v}=12.7 \mathrm{MPa} \\
\sigma_{h}=12.4 \mathrm{MPa} \\
\sigma_{H}=1.3 \sigma_{h}=16.12 \mathrm{MPa} \\
p_{w, 0}=4.7 \mathrm{MPa}
\end{gathered}
$$

where $\sigma_{v}$ is the vertical principal total stress, $\sigma_{h}$ is the minor horizontal principal total stress, $\sigma_{H}$ is the major horizontal principal total stress, and $p_{w, 0}$ is the initial pore water pressure. In that case, the initial threshold for the viscoplastic flow is $\alpha_{0}^{v p}=0.142$. Only this calibration is kept because it avoids an initial in situ creep deformation in the rock mass. A global calibration is realised for which the final values of the viscoplastic parameters are available in Table 4 and the correspondences with the experimental data are illustrated in Fig. 4. The parameters $A_{0}, \zeta$, and $n$ in Table 4 are involved in the viscoplastic flow rule defined in [29, 30].

\subsection{Strain localisation}

The main drawback of the homogeneous approach of the material behaviour is that it does not take into account any cracks or strain localisation effect. The onset and pattern of strain localisation in shear band is related to several material characteristics, which are mainly the softening parameters and the internal length scale. A softening plastic behaviour engenders the appropriate conditions for the onset of shear strain localisation which governs the postpeak behaviour. Thus, the calibration of the cohesion softening parameters would depend upon the structure of shear banding appearance in the CallovoOxfordian claystone. However, this information is rarely available for rocks especially for large-scale problems. Qualitative comparison between numerical and experimental results of compression test that involve shear bands in the 335 Callovo-Oxfordian claystone are available in [39, 5]. 
Table 4: Calibration of viscoplastic parameters for elasto-viscoplastic mechanical model.

\begin{tabular}{lllll}
\hline & Symbol & Name & Value & Unit \\
\hline Tests & & Laboratory & LAEGO & \\
description & $R H$ & Relative humidity & 90 & $\%$ \\
& $\sigma_{3}$ & Confining pressure & 12 & $M P a$ \\
& $\underline{q}$ & Stress deviator & $17,25.5,30.6$ & $M P a$ \\
\hline Global & $R_{c}$ & Uniaxial compressive strength & 21 & $M P a$ \\
parameters & $A$ & Internal friction coefficient & 2.62 & - \\
& $C_{s}$ & Cohesion coefficient & 0.03 & - \\
& $\beta^{v p}$ & Viscoplastic potential parameter & 1.1 & - \\
\hline Parameters for & $\alpha_{0}^{v p}$ & Initial threshold for the viscoplastic flow & 0.142 & - \\
$F_{0}^{v p}=0$ & $A_{0}$ & Reference fluidity & 700 & $s^{-1}$ \\
$($ in situ $)$ & $\zeta$ & Temperature parameter & $57 \times 10^{3}$ & $\mathrm{~J} / \mathrm{mol}$ \\
& $n$ & Creep curve shape parameter & 5.0 & - \\
& $B^{v p}$ & Viscoplastic hardening function parameter & $7.5 \times 10^{-3}$ & - \\
\hline
\end{tabular}



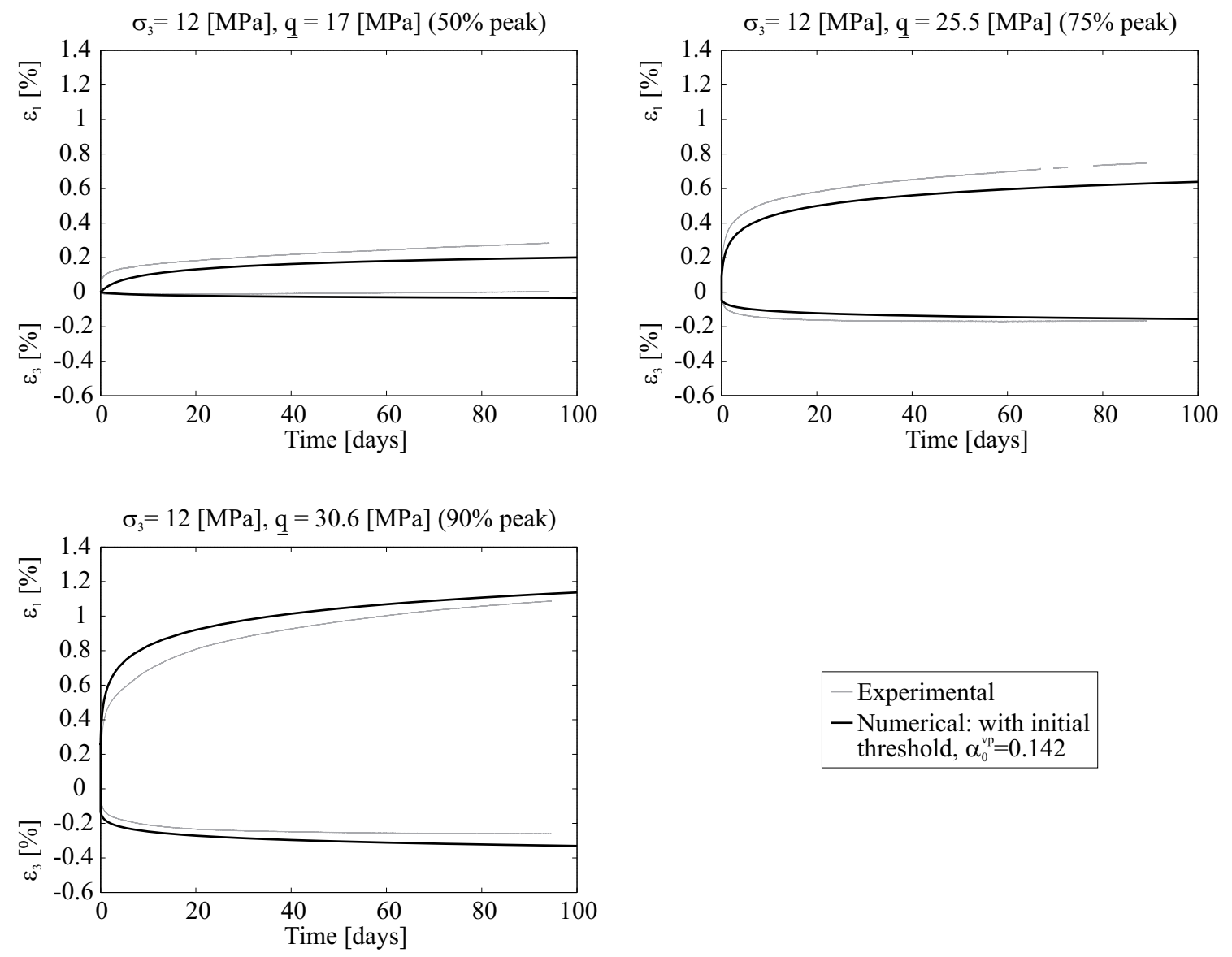

Figure 4: Fitting of creep tests for elasto-viscoplastic mechanical model. 
Strain localisation must still be triggered for the modelling which considers shear banding in a microstructure enriched medium. The values of the softening parameters are therefore adapted for the numerical application with the purpose of triggering strain localisation. The strain induced in the vicinity of the gallery during the drilling is lower than for compression experiments performed in laboratory. Therefore, the softening of the cohesion has to start earlier to allow the appearance of shear bands around the gallery. For the numerical modelling (section 4.3), a cohesion softening without delay is introduced with the softening parameters $\xi=c_{0} / c_{f}=10, B_{c}=0.003$, and $d e c_{c}=0$.

The second gradient mechanical law of Eq. 11 has also to be defined to correctly reproduce shear banding. The elastic constitutive parameter $D$ of this law is related to the internal length scale and represents the physical microstructure of the considered material. It should be estimated in relation to the thickness of the shear bands measured in the claystone, either on small-scale experiments or on a larger scale around the galleries. As mentioned for the evaluation of the softening parameters, information about shear banding thickness is rarely available in rocks on a large scale. Moreover, it has been demonstrated for any regularisation method that the thickness of the shear band should include several finite elements for a precise modelling of the post-localisation behaviour

355 [40]. For that purpose, a value of $D=5 k N$ is chosen because it allows to properly represent the shear banding with a good numerical precision within the shear bands.

\section{Modelling of the excavation damaged zone}

Among the different modelling cases realised in the benchmark "Transverse action", only some of them are exposed in the following modelling. The purpose is to highlight the effects of anisotropy, viscosity, and shear banding appearance around a gallery, both during and after the drilling. The numerical modelling considers the drilling of the GCS gallery of the Andra's Underground Research Laboratory (URL), with hydro-mechanical coupling and a flexible liner on the 


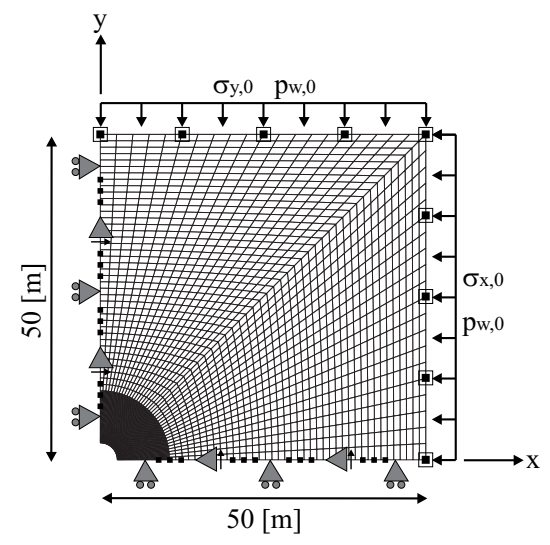

$\begin{aligned} & \text { Drained boundary with } \\ & \text { constant pore water } \\ & \text { pressure }\left(\mathrm{p}_{\mathrm{w}, 0)}\right) \\ & \text { Constant total stress } \\ & \left(\sigma_{\mathrm{x}, 0}, \sigma_{\mathrm{y}, 0)}\right) \\ & \text { Constrained } \\ \text { displacement } & \text { dispendicular to } \\ \text { perpend } & \text { the boundary } \\ & \text { Constrained normal } \\ \Delta & \text { derivative of the radial } \\ & \text { displacement } \\ \ldots & \text { Impervious boundary }\end{aligned}$

Figure 5: Numerical model and boundary conditions used for the modelling of a gallery excavation. water flow) and no normal displacements. Moreover, for the modelling that 
involves strain localisation (section 4.3) and the second gradient model (defined in sections 2.1.2 and 2.2.2, the normal derivative of the radial displacement 385 is equal to zero along the symmetry axes [3]. This supplementary kinematic condition is required because of the gradient terms included in the expression of the equilibrium equation of the second gradient model (Eq. 4).

The drilling of the gallery is realised by decreasing the total radial stress on the gallery wall following a uniform deconfinement curve adapted from the convergence-confinement method [1]. For the benchmark "Transverse action", the complete excavation is performed in a total of 28 days with the excavation front crossing the studied section after 14 days. Among the different possible supports at gallery wall after the excavation, only a flexible liner is considered (no rigid liner). It is modelled by maintaining a constant total radial stress of $395 \quad 0.3 \mathrm{MPa}$ at gallery wall after the drilling. This radial stress corresponds to the 25 th day in the deconfinement curve after which the radial stress is imposed constant.

Concerning the pore pressure at gallery wall, it decreases rapidly from its initial value to zero when the drilling front crosses the studied gallery section. After the excavation, a zero constant pore water pressure is maintained on the gallery wall. It means that the air inside the gallery remains fully saturated with water vapour after the excavation, which corresponds to an air relative humidity of $100 \%$ by Kelvin's law. It consists of a classical flow boundary condition at gallery wall assuming an instantaneous hydraulic equilibrium between the liquid water inside the porous rock and the water vapour of the cavity air. However, this imposition at gallery wall does not correspond to the air ventilation usually realised inside underground galleries. The air ventilation could be taken into account to better represent the hydraulic transfer around the galleries [2]. Furthermore, unsaturated conditions are also considered but their impact is limited because the claystone remains almost saturated in the absence of gallery air ventilation.

More information about the deconfinement curves for the radial stress and the pore water pressure can be found in the description of the benchmark 


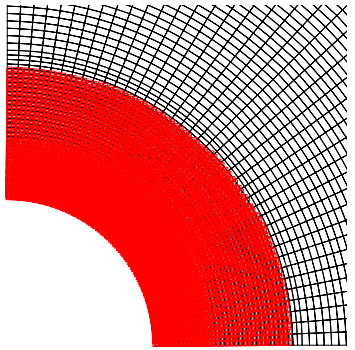

(a)

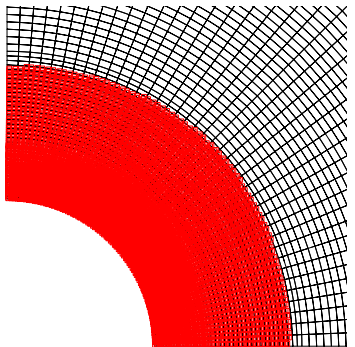

(b)

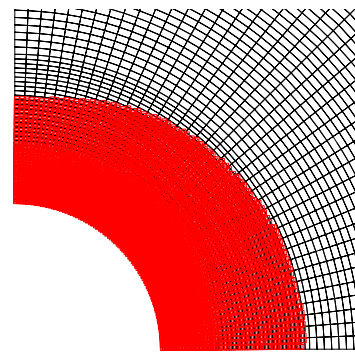

(c)

Figure 6: Plastic zone at the end of excavation for modellings without viscosity and with several mechanical behaviours: (a) isotropic, (b) anisotropic elastic, and (c) anisotropic elastoplastic.

"Transverse action".

\subsection{Classical medium without strain localisation}

The modelling of the gallery excavation is firstly realised for a classical medium (section 2.1.1) without considering strain localisation, neither cohesion softening $\left(\xi=c_{0} / c_{f}=1\right)$. The latter will be considered for the modelling involving shear bands in section 4.3 . However, the hardening of the friction angles and the difference between compressive and extensional characteristics are considered. The different components of the material behaviour will be added step by step to highlight their influences.

For an isotropic mechanical material behaviour without viscosity $(E=$ $\left.4 G P a, \nu=0.3, b=0.6, k=4 \times 10^{-20} \mathrm{~m}^{2}, c=4.2 M P a, \epsilon^{v p}=0\right)$, the plastic zone appearing around the gallery at the end of excavation ( 25 days) is exposed in Fig. 6 (a). The Gauss integration points under plastic loading are represented with red squares and the other integration points are under elastic regime. One can observe that plasticity develops around the gallery during the excavation with a quasi-circular extension of about 0.9 gallery radius $(2.35 \mathrm{~m})$ in the rock. The extension is slightly more important in the horizontal direction because of the hydraulic anisotropy which is taken into account for the intrinsic water permeability, even if the mechanical anisotropy is not considered. 


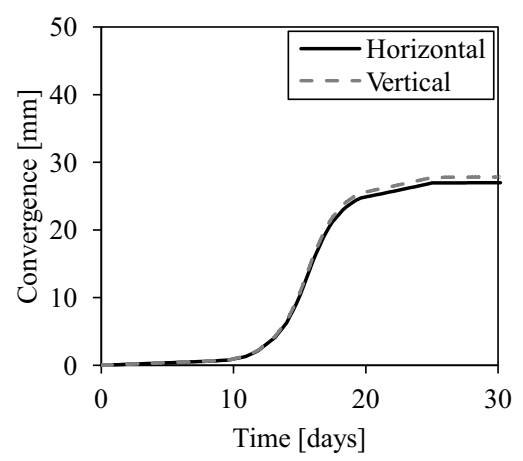

(a)

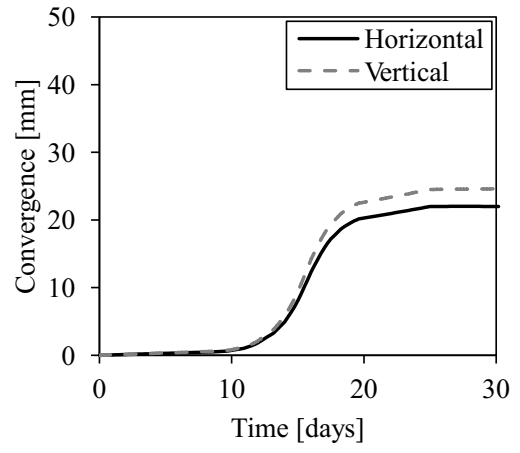

(b)

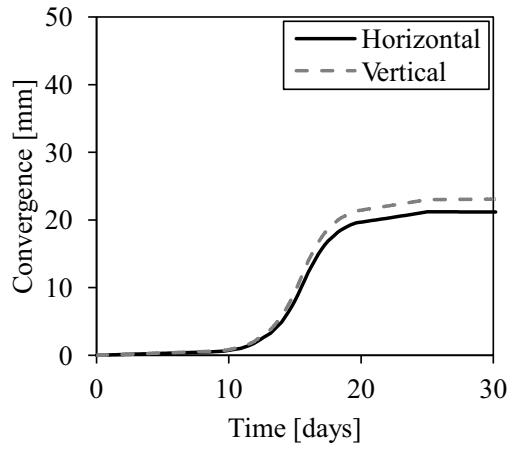

(c)

Figure 7: Gallery convergences during the excavation for modellings without viscosity and with several mechanical behaviours: (a) isotropic, (b) anisotropic elastic, and (c) anisotropic elasto-plastic.

The diametrical convergence of the gallery during the excavation is illustrated in Fig. 7 (a) for both the horizontal and vertical directions. The convergences are almost identical; the slight difference is once again related to the anisotropy of the intrinsic water permeability. The convergences are constant in the long term because the material viscosity is not taken into account so far.

The anisotropic properties of the material mechanical behaviour, with horizontal isotropic bedding planes, can also be considered. Let us first consider 440 the anisotropy of the elastic characteristics of Table 2. The plastic zone and the convergences are illustrated in Fig. 6 (b) and Fig. 7(b). It can be seen that the shape of the plastic zone is almost not affected by this anisotropy. The convergences reduce and are anisotropic with a more important convergence in the vertical direction. The anisotropy of the elastic moduli that has been added ${ }_{445} E_{\|}>E_{\perp}$ leads to smaller deformation in the horizontal direction than in the vertical direction. Moreover, the material is globally stiffer which causes a slight decrease of both convergences.

Secondly, the anisotropic characteristics of the plastic behaviour is also added with the microstructure fabric tensor for the cohesion (Eq. 7 and section 3.3.3. 450 The plastic zone and the convergences are available in Fig. 6(c) and Fig. 7 (c). The extent of the plastic zone in the vertical direction is slightly reduced because 


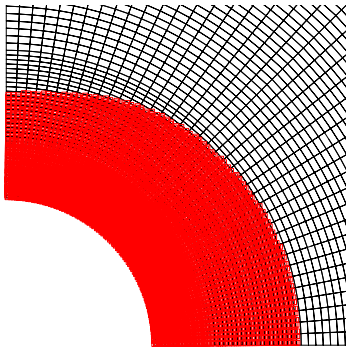

(a)

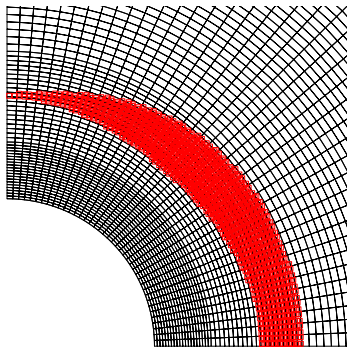

(b)

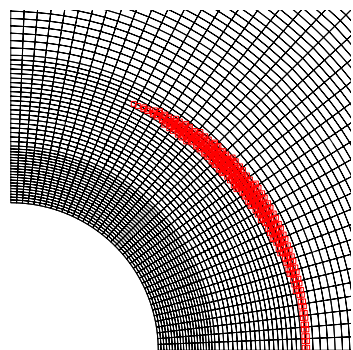

(c)

Figure 8: Evolution of the plastic zone after the excavation for an anisotropic rock with viscosity: (a) end of excavation, (b) 30 days, and (c) 60 days of calculation.

the cohesion increases above and below the gallery during the excavation. The cohesion increase is less important in the horizontal direction. This difference of cohesion increase in the two directions also explains the slight reduction of fractures [2]. 


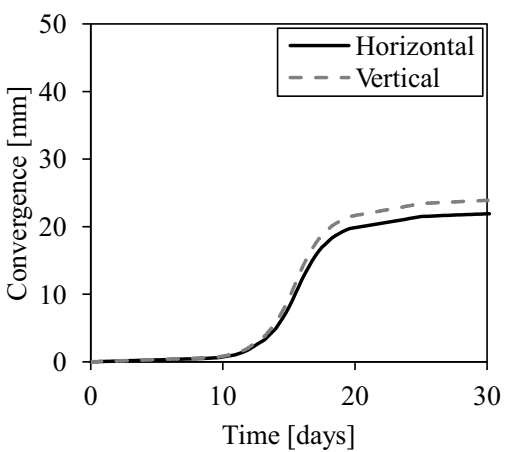

(a)

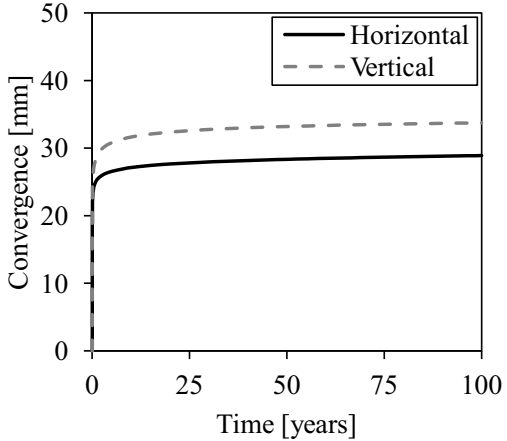

(b)

Figure 9: Gallery convergences for an anisotropic rock with viscosity: (a) during the excavation and (b) in the long term.

The diametrical gallery convergences are detailed in Fig. 9, for both short and long terms. The viscosity effects are mainly apparent in the long term, with an increase of the convergence after the gallery excavation. It can be noticed that the anisotropy of the convergence also increases in the long term due to the material anisotropy.

Both convergences do not match experimental in situ measurements of convergence of the GCS a gallery. For the different sections of convergence measurement (GCS-OHZ170B to G), the experimental results indicate that the convergence is anisotropic. The major convergence is measured in the horizontal direction (Fig. 13) which corresponds to the location of the fractures around the gallery [1]. Furthermore, experimental measurements also indicate that the gallery convergence increases in the long term but in a much larger amplitude, especially in the horizontal direction. Neither the anisotropy of the convergence nor its increase in the long term is captured by the numerical modelling, even if the material viscosity is considered. Consequently, considering the material anisotropy and a viscosity based on laboratory creep tests are not sufficient to reproduce the observed shape of the damaged zone and the gallery convergence. 


\subsection{Microstructure medium with strain localisation}

The previous modelling with a classical approach has highlighted that taking into account the anisotropic properties of the material is not sufficient to reproduce experimental observations related to the excavation damaged zone. Considering the representation of material discontinuities seems necessary to improve the excavation damaged zone modelling. Hereafter, it is proposed to model the shear fractures by considering shear strain localisation bands as a precursor to material rupture. A strain softening behaviour is required to engender the conditions for the onset of strain localisation. It is introduced through cohesion softening in the yield surface of the plastic material behaviour. Moreover, the regularisation method described in sections 2.1 .2 and 2.2 .2 is used to properly model the strain localisation. Let us recall that, for the modelling involving shear banding, the values of the softening parameters and of the second gradient elastic modulus are defined in section 3.5 .

A modelling of the EDZ with shear banding in the Callovo-Oxfordian claystone has been performed in [5]. The numerical results highlight how the material anisotropy influences the strain localisation appearance during gallery excavation in case of an isotropic initial stress state. When considering the Callovo-Oxfordian claystone, its anisotropic stress state must also be taken into account because it influences the fracturing structure. For strain localisation approach, the stress anisotropy has been considered for a gallery oriented parallel to the minor horizontal principal stress $\sigma_{h}$ in [3, 42] but not for a gallery parallel to the major horizontal principal stress $\sigma_{H}$. For a gallery parallel to $\sigma_{h}$ and for an isotropic mechanical behaviour of the rock, the results in [3,43] indicate that the initial stress state anisotropy in the gallery section controls the fracturing appearance and pattern. For a gallery parallel to $\sigma_{H}$, the major stress is in the axial direction and the stress state is quasi-isotropic in the gallery section $\left(\sigma_{h} \approx \sigma_{v}\right)$. It requires an anisotropic mechanical behaviour of the material to exhibit strain localisation during the excavation. The modelling detailed hereafter includes the major horizontal principal stress in the longitudinal (axial) 520 direction of the gallery. 


\subsubsection{Shear banding pattern}

The evolutions of the total deviatoric strain and plastic zone during the drilling are illustrated in Fig. 10 (a) and (b). The plastic zone is represented by the plastic loading integration points (red squares) and the Von Mises' equivalent deviatoric total strain, i.e. total deviatoric strain, is defined as:

$$
\hat{\epsilon}_{e q}=\sqrt{\frac{2}{3} \hat{\epsilon}_{i j} \hat{\epsilon}_{i j}}
$$

where $\hat{\epsilon}_{i j}=\epsilon_{i j}-\frac{\epsilon_{k k}}{3} \delta_{i j}$ is the deviatoric part of the total strain field $\epsilon_{i j}$. In Fig. 10, one can observe the appearance and the development of the shear bands before the end of the excavation ( 25 days). In fact, the complete formation of shear bands occurs between 19.6 and 25 days that correspond to a radial total stress at gallery wall of $0.84 M P a$ and $0.3 \mathrm{MPa}$, respectively. The strain localisation develops preferentially in the horizontal direction. This strain localisation development is governed by the cross-anisotropic plastic mechanical characteristics of the claystone [5].

To better understand the preferential development of the EDZ in the horizontal direction, let us consider the cohesion around the gallery at the beginning of the strain localisation appearance (19.6 days) in Fig. 10 (c). During the excavation, the radial stress decreases at gallery wall and the orthoradial stress increases. It engenders a loading that tends to be parallel to the bedding planes in the vertical direction and perpendicular to the bedding planes in the horizontal direction. Above and below the gallery, the cohesion increases due to the loading evolution which prevents the development of shear bands in this direction. In the horizontal direction, the cohesion slightly increases but less than in the vertical direction because of the stress component in the axial direction of the gallery. At $45^{\circ}$ with the horizontal, the cohesion remains low. Consequently, the shear bands start appearing where the resistance of the material is the lowest (at about $45^{\circ}$ ) and the development of the strain localisation zone propagates in the horizontal direction during the rest of the drilling (from 19.6 to 25 days). In the shear bands, the cohesion decreases because of softening ${ }_{545}$ plasticity (Fig. 10 (c)). 

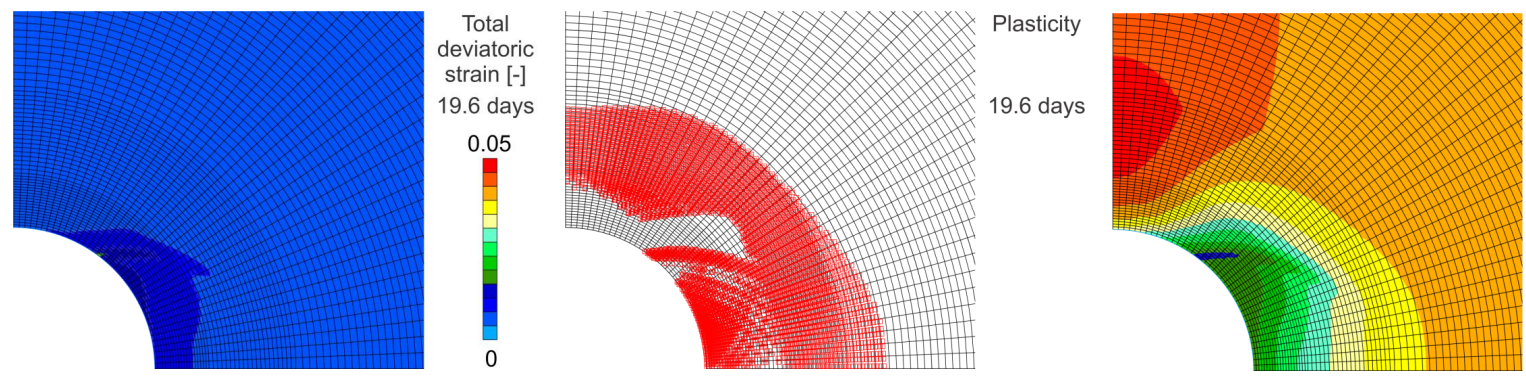

Cohesion [MPa]

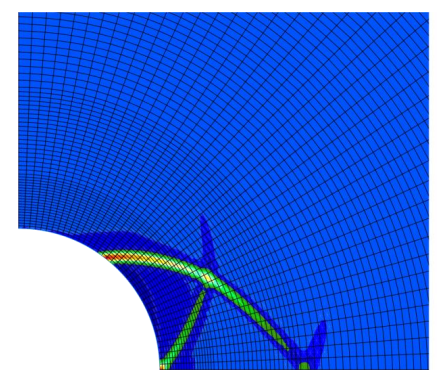

Total strain [-] 25 days

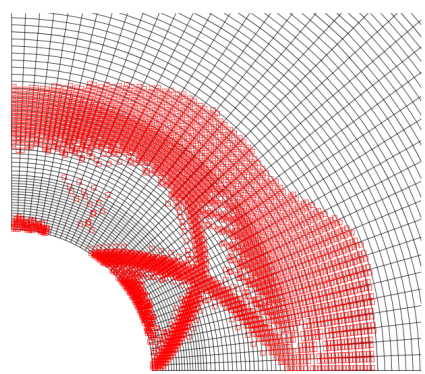

Plasticity

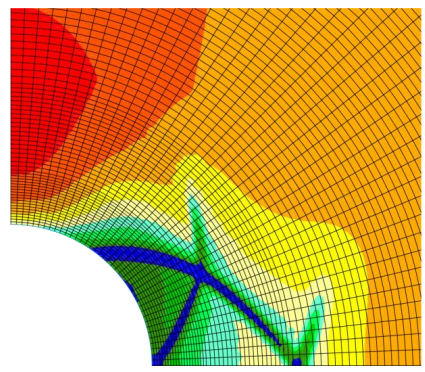
19.6 days

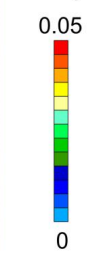

Total
deviatoric

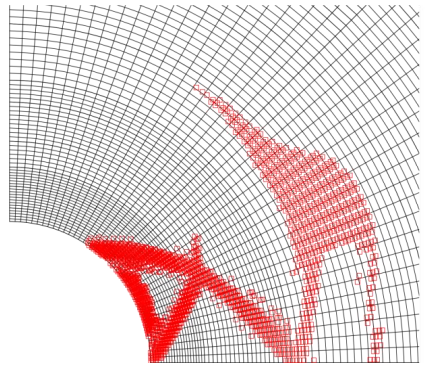

Plasticity
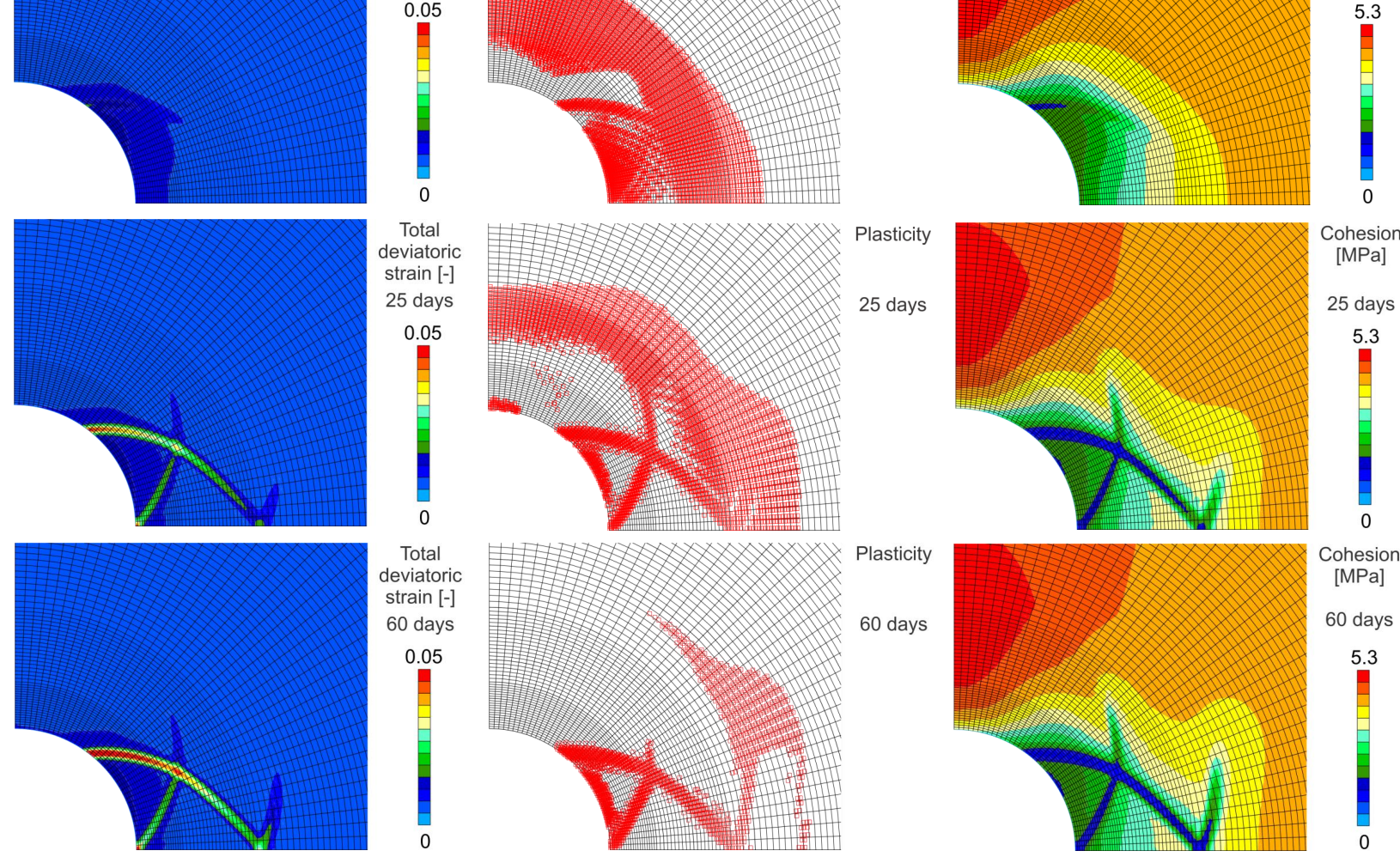

strain [-]

60 days

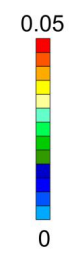

Total
deviatoric
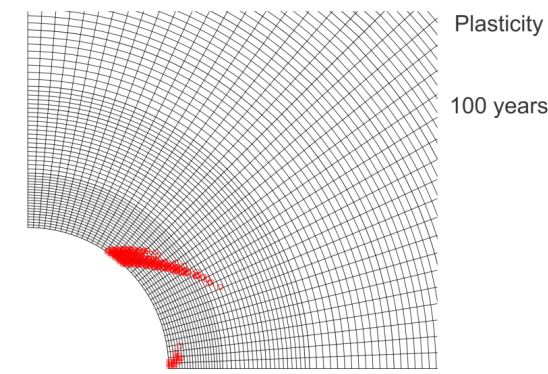

(b)

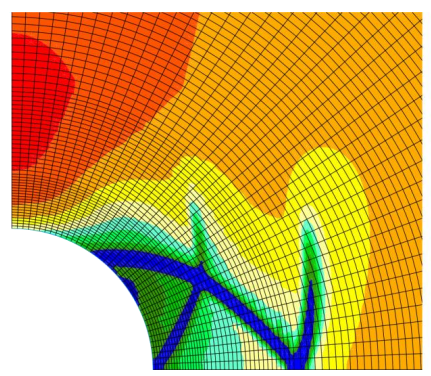

Cohesion

$[\mathrm{MPa}]$

25 days

5.3

60 days $[\mathrm{MPa}]$

60 days

Cohesion [MPa]

100 years

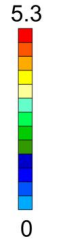

(c)

Figure 10: Development of shear bands around a gallery parallel to the major horizontal principal stress: (a) total deviatoric strain, (b) plastic zone, and (c) cohesion. 


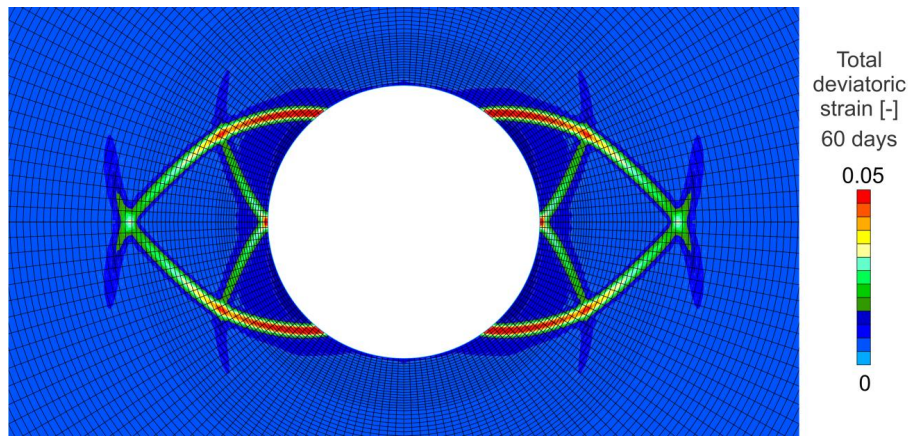

Figure 11: Shear banding around a gallery parallel to the major horizontal principal stress.

After the drilling, the pattern of shear bands does not evolve significantly and an increase of the deformation is observed within the shear bands because of plastic strain localisation. Furthermore, the material becomes progressively elastic close to the gallery (Fig. 10 (b) for 60 days and 100 years of calculation) but the process of elastic unloading takes more time than for the previous modelling (Fig. 8). The latter is related to the cohesion softening in the shear bands (Fig. 10 (c)) which maintains the rock in plastic regime. The decrease of cohesion opposes the effect of drainage and delays the elastic unloading in the strain localisation zones.

555 The full shear band pattern is recomposed by symmetry in Fig. 11. The observed shape is in good agreement with experimental measurements of the shear fracture pattern and extent around the gallery [2]. The extent of the numerical shear banding zone is compared in Table 5 to the in situ measurements of shear and tensile fractures around the gallery [2]. It indicates a satisfactory agreement. The shear banding zone extent lies between the mixed (shear and tensile) fracture zone and the shear fracture zone.

\subsubsection{Gallery convergence and rock deformation}

The diametrical convergence of the gallery is illustrated in Fig. 13 and compared to experimental measurements of the GCS gallery convergence [1]. Because the convergence measurement sections are installed after the excavation front, the beginning of the measurements corresponds to the 14th day of the 

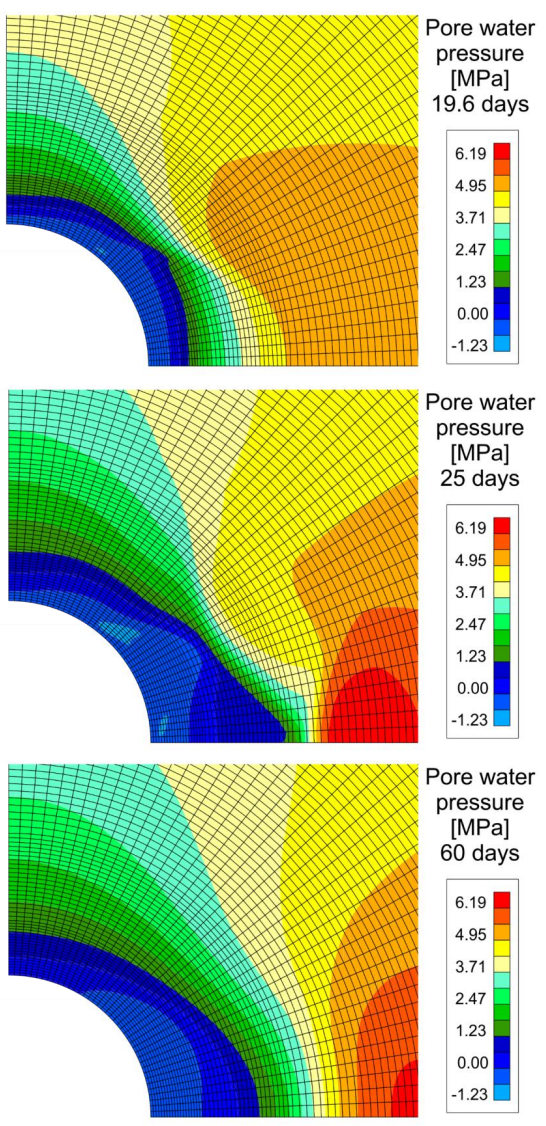

Pore water pressure
[MPa]

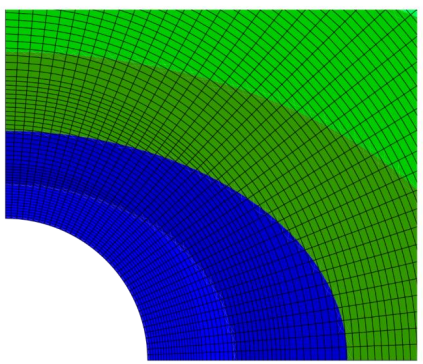

60 days

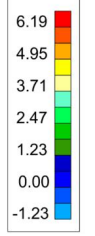

Pore water

pressure

[MPa]

\begin{tabular}{|c|}
\hline 6.19 \\
4.95 \\
3.71 \\
2.47 \\
1.23 \\
0.00 \\
-1.23 \\
\hline
\end{tabular}

Figure 12: Evolution of pore water pressure around a gallery parallel to the major horizontal principal stress. 
Table 5: Comparison between the thickness of the numerical shear strain localisation zone and the extents of the fractured zones around a gallery (GCS) parallel to the major horizontal principal stress.

\begin{tabular}{llll}
\hline Zone & Horizontal left $[\mathrm{m}]$ & Horizontal right $[\mathrm{m}]$ & Vertical $[\mathrm{m}]$ \\
\hline Numerical shear banding & 3 & 3 & 0 \\
Mixed fractures & 1.4 & 1.4 & $<0.5$ \\
Shear fractures & 4.7 & 4.5 & $<0.5$ \\
\hline
\end{tabular}

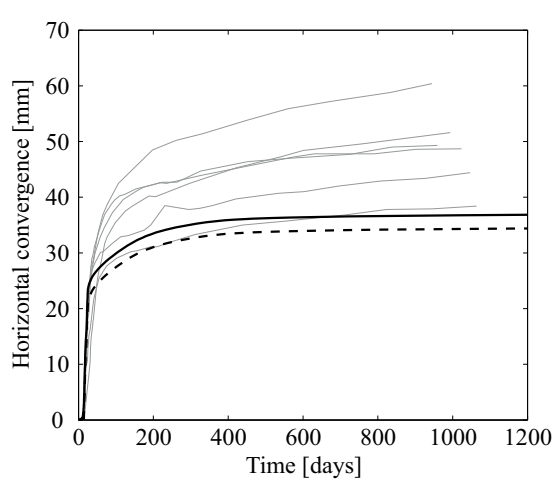

(a)

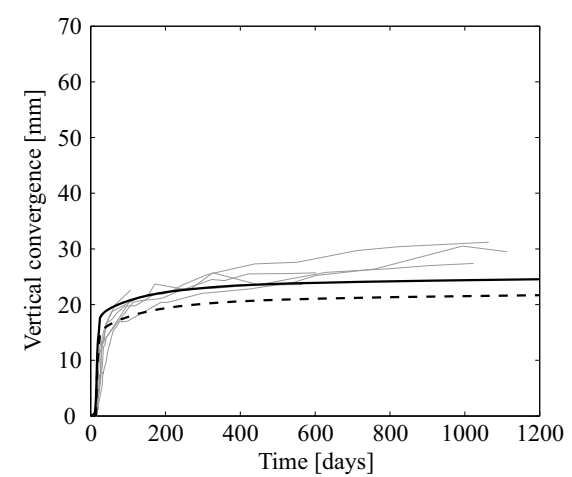

(b)

Figure 13: Evolution of the convergence of a gallery parallel to the major horizontal principal stress in (a) horizontal and (b) vertical directions.

numerical modelling. In fact, the excavation front crosses the studied gallery section after 14 days in the numerical modelling as described in the deconfinement curve used in the present benchmark. Two numerical convergences are detailed in Fig. 13. one from the beginning of the calculation (day 0) that includes the rock deformation developing before the excavation front crosses the studied section, and another from the excavation front (day 14) that corresponds only to the gallery convergence. The numerical modelling captures correctly the convergences for both directions; nonetheless, the viscosity effects seems to remain limited in the long term.

In the Andra's URL, displacement measurements in the rock mass around galleries are also realised in addition to the measurements of diametrical con- 
vergence. They are performed with borehole extensometers drilled from the considered gallery or from a pre-existing gallery. These extensometers record the radial displacements between the gallery wall (tip of the extensometer) and the anchors located at different depths in the rock formation. These measurements permit to quantify the displacement and the deformation of the rock.

The first borehole extensometer that is studied was drilled in the context of the mine-by experiment of the GCS drift. This experiment is a state-of-the-art project that aims to characterise the various impacts of underground drilling on the rock [1]. Before the GCS drift was excavated, different instrumented boreholes were drilled from surrounding galleries towards the location of the future drift. These boreholes contain extensometers, pore pressure sensors, and inclinometers. Among them, the borehole extensometer OHZ1501 was drilled horizontally from the GAT gallery, located $30 \mathrm{~m}$ away from the GCS location. The different anchors are set up from the GAT drift wall up to $30 \mathrm{~m}$ in the rock as illustrated in Fig. 14. The measurements of radial displacements between the GAT drift wall and the anchors are available in [1]. They can be easily transformed in radial displacements between the GED drift wall and the anchors by assuming that the anchor located at $30 \mathrm{~m}$ depth from the GAT drift wall corresponds to the GED drift wall. The second borehole extensometer that is considered is the OHZ1707. It is drilled vertically upward from a gallery measurement section that is installed inside the GCS gallery during its drilling progress, which means just after the excavation front. The different anchors are 600 set up from the GCS drift wall up to a depth of about $30 \mathrm{~m}$ in the claystone (Fig. 14 (b)).

In the numerical modelling, the relative radial displacement between any location and the gallery wall corresponds to:

$$
\Delta u_{r}=u_{r}-u_{r}^{\Gamma}
$$

where $u_{r}$ and $u_{r}^{\Gamma}$ are the current radial displacements of a considered material point and of the gallery wall for the same orientation. The relative radial displacements are compared to the experimental measurements for both boreholes 


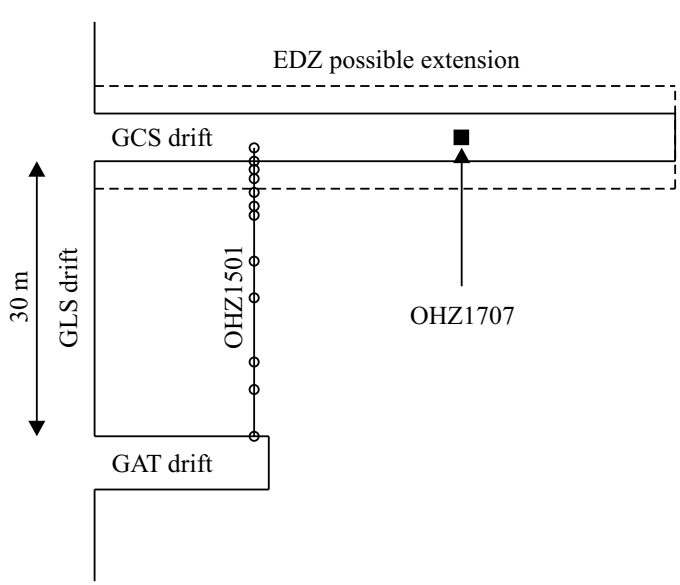

(a)

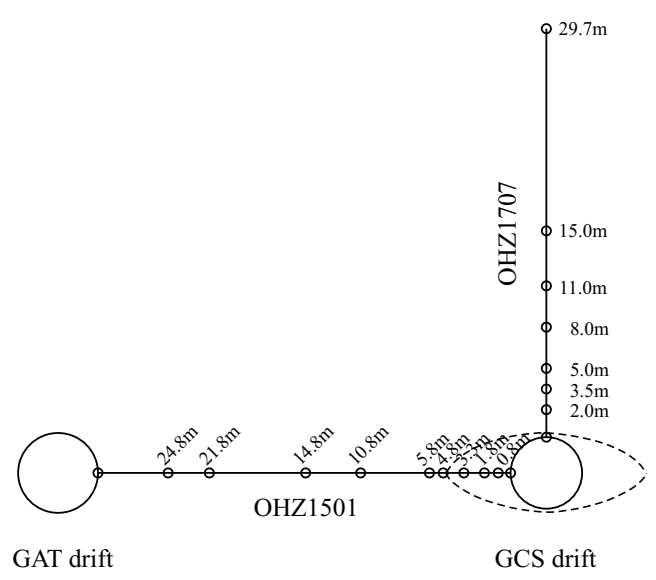

(b)

Figure 14: Positions of the extensometer boreholes around the GCS drift: (a) in a horizontal plane and (b) in the cross section of the drift with the extensometers and the radial distances of the anchors to the gallery wall.

in Fig. 15 where:

$$
\begin{aligned}
& \Delta u_{x}=u_{x}-u_{x}^{\Gamma} \\
& \Delta u_{y}=u_{y}-u_{y}^{\Gamma}
\end{aligned}
$$

and the different distances from the gallery wall correspond to the anchors of the extensometers. The numerical results are detailed only after the excavation front crosses the studied gallery section: from 14 days in Fig. 15 (a) and from 17.4 borehole, in fact it was drilled $1.2 \mathrm{~m}$ behind the gallery front, which corresponds to 17.4 days in the convergence confinement curve described in the benchmark "Transverse action". As for the gallery convergence, the displacements are satisfactorily reproduced unless for the long-term horizontal displacements.

\subsubsection{Viscosity effect}

The elasto-viscoplastic model can be tested for different sets of viscoplastic parameters to highlight their influences on deformation and gallery conver- 
(a)
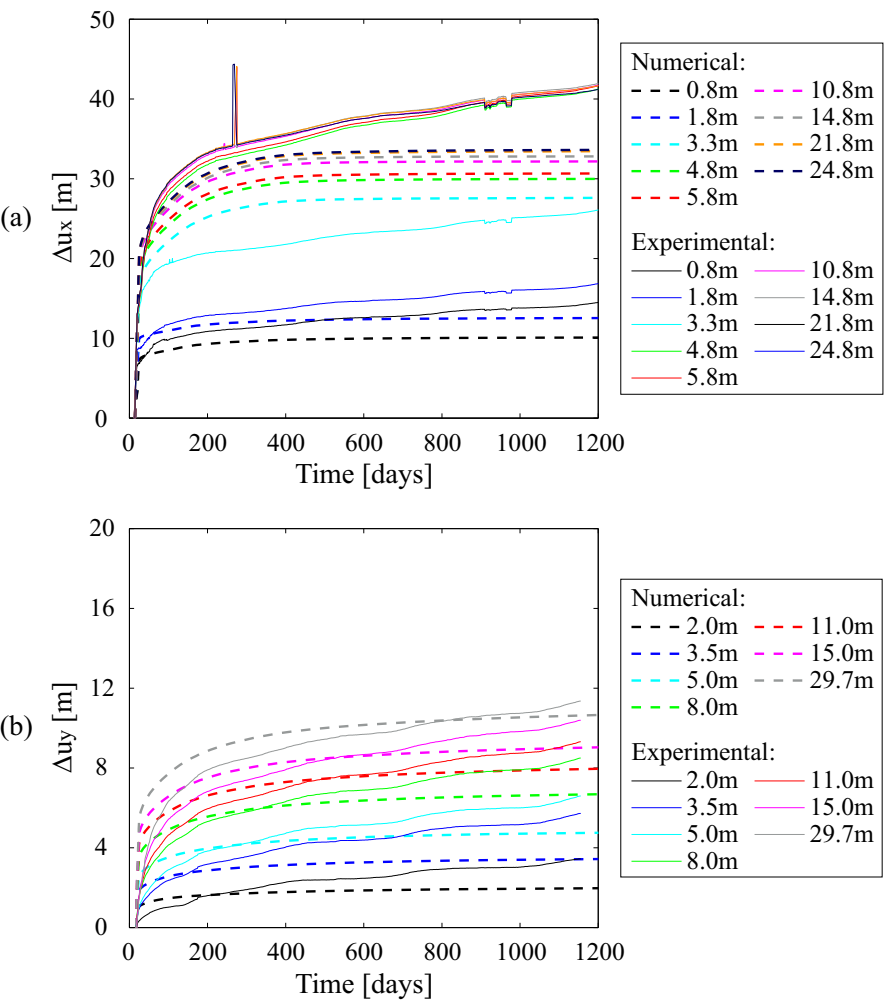

Figure 15: Evolution of relative radial displacements to gallery wall for two borehole extensometers: (a) OHZ1501 horizontal and (b) OHZ1707 vertical upward. 
gence. In fact, the increases of convergence and relative displacements in the long term could be better reproduced by adjusting the viscoplastic parameters of Table 4. These parameters come from experimental data fitting based on laboratory creep tests (section 3.4 that last a hundred days, not a thousand days like the in situ experimental measurements around the underground gallery. Different sets of viscoplastic parameters are tested and their results in terms of gallery convergence are illustrated in Fig. 16. They are compared to experimental measurements from the 14th day of the numerical excavation.

Firstly, comparing the results with the viscoplastic parameters based on the creep tests to the results without taking into account material viscosity indicates that these parameters generate only a limited long-term deformation. In fact, the numerical curves for both convergences are relatively close in the long term. The viscoplastic parameters must be adapted to increase the longterm deformation and have a better reproduction of the gallery convergence. This modification is consistent with a possible coupling between plasticity (or damage) and viscosity, even if this coupling is not actually reproduced.

For the considered viscoplastic mechanism, increasing the parameter $B^{v p}$ will slow down the evolution of the function $\alpha^{v p}$ (Eq. 10) as well as the hardening of the viscoplastic loading surface (Eq.9). This will have the effect of increasing the viscoplastic flow $F^{v p}$ and deformation $\epsilon_{i j}^{v p}$. Multiplying by ten this parameter $\left(B^{v p}=7.5 \times 10^{-2}\right)$ leads to larger long-term deformations and to a better match with the in situ measurements of convergences, in both horizontal and vertical 635 directions (Fig. 16). The convergences from the beginning of the calculation (day 0) are also displayed in Fig. 16 for this last set of viscoplastic parameters.

The relative radial displacements for the two borehole extensometers are reproduced with this new set of viscoplastic parameters. Fig. 17]illustrates that the prediction of the horizontal displacements is improved, but the prediction of 640 the vertical displacements is deteriorated. Vertical displacements were already well reproduced in Fig. 15 (b) so increasing the viscosity can only deteriorate the agreement with the experimental measurements of the vertical borehole. Nevertheless, this borehole extensometer concerns only one measurement section 


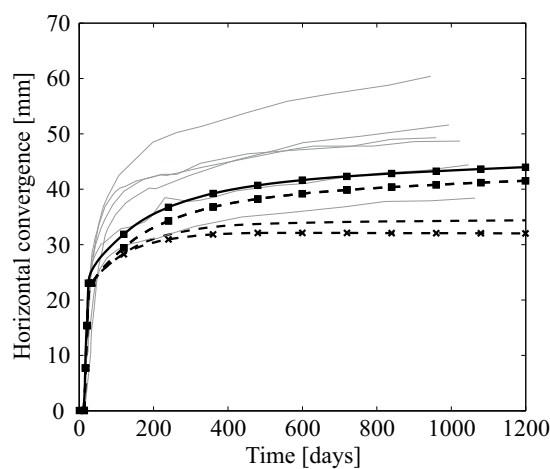

(a)

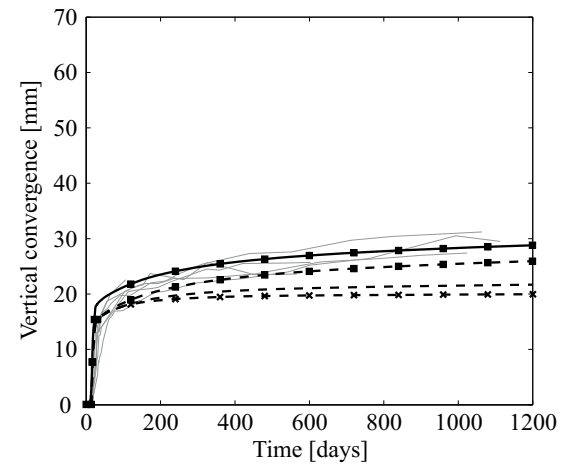

(b)

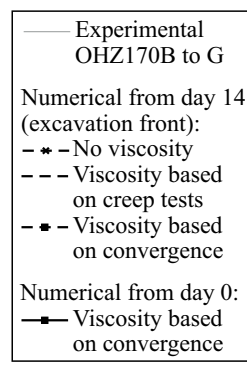

Figure 16: Evolution of the convergence of a gallery parallel to the major horizontal principal stress for different sets of viscoplastic parameters in (a) horizontal and (b) vertical directions.

in the GCS gallery while the convergence measurements in Fig. 16 are related

${ }_{645}$ to several measurement sections, which make them more reliable in a general sense.

\section{Conclusions}

The benchmark "Transverse action" conducted by Andra, the French national radioactive waste management agency, consists in developing and calibrating numerical models for the Callovo-Oxfordian claystone with the purpose of using them to model underground structures. These structures are underground galleries which are modelled by the reproduction of the drilling phase. The proposed constitutive model is a cross-anisotropic and elasto-viscoplastic model which is calibrated based on experimental results obtained in laboratory, on small-scale samples. The excavation of a gallery is modelled at large scale with finite element method. The numerical modelling takes into account the development of shear bands to represent macroscale shear fractures.

In the presented modelling approach, the focus is on the contributions of material anisotropy, of viscosity, and especially of shear bands to represent the rock behaviour and the shear fractures. The excavation of a gallery oriented along the major horizontal principal stress of the rock and having a quasi- 
(a)
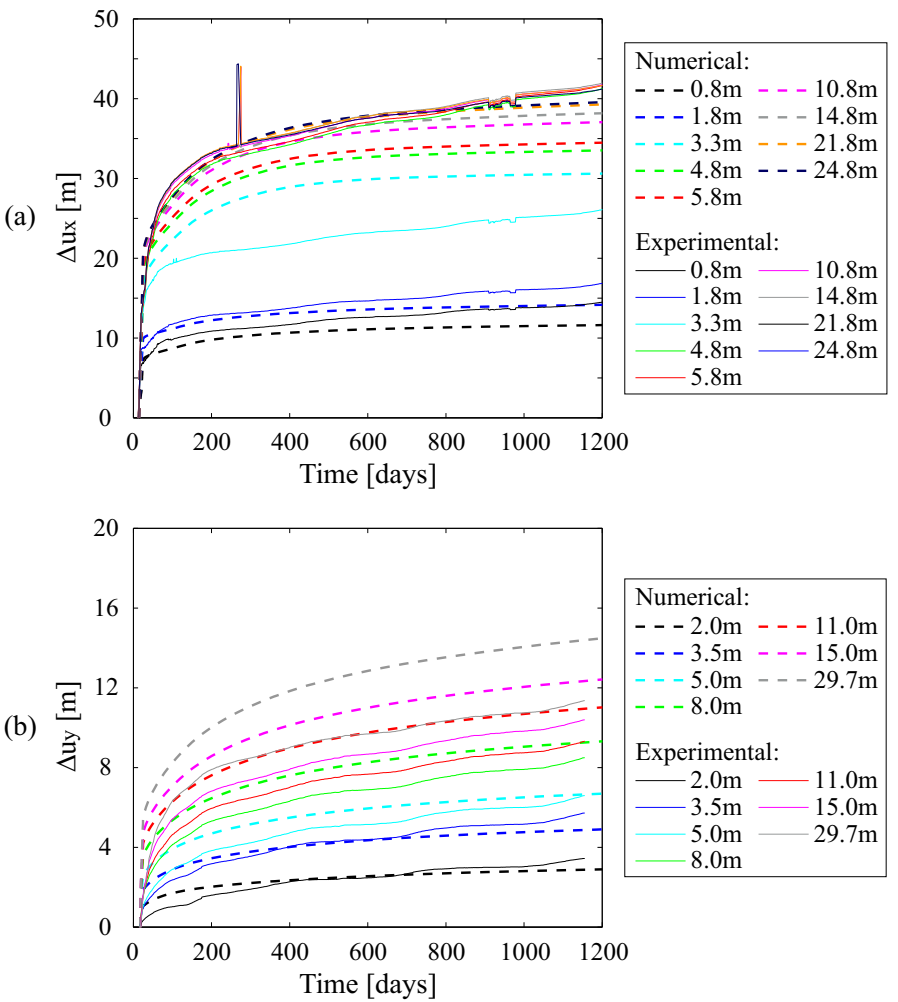

Figure 17: Evolution of relative radial displacements to gallery wall with improvement of the viscoplastic parameters, for two borehole extensometers: (a) OHZ1501 horizontal and (b) OHZ1707 vertical upward. 
isotropic stress state in its section is studied. A classical approach with an anisotropic model is used at first. The numerical results indicate that, even if the material viscosity is taken into account, neither the Excavation Damaged

665 Zone (EDZ) nor the gallery convergence are well reproduced with this approach. For instance, the anisotropy of the diametrical convergence of the gallery can not be reproduced by such continuous model.

Modelling shear banding in the rock around the gallery is an important aspect that allows to represent the shear fractures with strain discontinuities and that leads to a better reproduction of the EDZ in the claystone. The shear strain localisation bands are properly represented by a regularisation method involving a microstructure medium. The shear bands correctly reproduce the extent of the macroscale shear fractures measured in the rock. For the considered gallery, the preferential development of the shear bands in the horizontal direction is explained by the plastic anisotropy of the material. In fact, the strain localisation zone develops preferentially in the direction of lower material resistance.

The gallery convergence and rock deformation are also well reproduced. The convergence anisotropy is reproduced thanks to the shear banding appearance 680 with a larger convergence in the direction of the location of the shear bands. Furthermore, creep deformations have been introduced with a viscoplastic mechanism which allows to reproduce the increase of convergence in the long term.

\section{Acknowledgements}

The authors are grateful for financial supports from the FRIA-F.R.S.-FNRS, French national radioactive waste management agency.

\section{References}

[1] G. Armand, A. Noiret, J. Zghondi, D. M. Seyedi, Short- and long-term behaviors of drifts in the Callovo-Oxfordian claystone at the Meuse/Haute- 

(2013) 221-230.

[2] G. Armand, F. Leveau, C. Nussbaum, R. de La Vaissiere, A. Noiret, D. Jaeggi, P. Landrein, C. Righini, Geometry and properties of the excavation-induced fractures at the Meuse/Haute-Marne URL drifts, Rock Mech Rock Eng 47 (1) (2014) 21-41.

[3] B. Pardoen, S. Levasseur, F. Collin, Using Local Second Gradient Model and Shear Strain Localisation to Model the Excavation Damaged Zone in Unsaturated Claystone, Rock Mech Rock Eng 48 (2) (2015) 691-714. doi:10.1007/s00603-014-0580-2.

[4] J. Desrues, G. Viggiani, Strain localization in sand: an overview of the experimental results obtained in Grenoble using stereophotogrammetry, Int J Numer Anal Meth Geomech 28 (4) (2004) 279-321.

[5] B. Pardoen, D. M. Seyedi, F. Collin, Shear banding modelling in cross(1) anisotropic rocks, Int J Solids Struct 72 (2015) 63-87. doi:10.1016/j. ijsolstr.2015.07.012.

[6] R. Charlier, Approche unifiée de quelques problèmes non linéaires de mécanique des milieux continus par la méthode des éléments finis (grandes déformations des métaux et des sols, contact unilatéral de solides, conduction thermique et écoulements en milieu poreux), Ph.D. thesis, Faculté des Sciences Appliquées, Université de Liège, Belgium (1987).

[7] M. A. Biot, General theory for three-dimensional consolidation, J Appl Phys 12 (2) (1941) 155-164.

[8] F. Collin, B. Pardoen, Excavation damaged zone modelling in claystone with coupled second gradient model, in: Q. Yang, J. M. Zhang, H. Zheng, Y. Yao (Eds.), Constitutive Modeling of Geomaterials, Springer Series in Geomechanics and Geoengineering, Springer, Berlin Heidelberg, 2013, pp. 313-317. doi:10.1007/978-3-642-32814-5_42. 
[9] B. Pardoen, S. Levasseur, F. Collin, Using shear strain localisation to model the fracturing around gallery in unsaturated Callovo-Oxfordian claystone,

[10] F. Collin, S. Levasseur, R. Chambon, Numerical post failure methods in

[11] R. Toupin, Elastic materials with couple-stresses, Arch Ration Mech An 11 (1) (1962) 385-414.

[12] R. D. Mindlin, Micro-structure in linear elasticity, Arch Ration Mech An 16 (1) (1964) 51-78.

[13] R. Chambon, D. Caillerie, T. Matsushima, Plastic continuum with microstructure, local second gradient theories for geomaterials : localization studies, Int J Solids Struct 38 (46-47) (2001) 8503-8527.

[14] F. Collin, R. Chambon, R. Charlier, A finite element method for poro mechanical modelling of geotechnical problems using local second gradient models, Int J Numer Meth Engng 65 (11) (2006) 1749-1772.

[15] W. Ehlers, W. Volk, On theoretical and numerical methods in the theory of porous media based on polar and non-polar elasto-plastic solid materials, Int J Solids Struct 35 (34-35) (1998) 4597-4617.

[16] A. E. H. Love, A treatise on the mathematical theory of elasticity, 4th Edition, Cambridge University Press, 1927.

[17] J. Graham, G. T. Houlsby, Anisotropic elasticity of a natural clay, Géotechnique 33 (2) (1983) 165-180.

[18] A. H. D. Cheng, Material coefficients of anisotropic poroelasticity, Int J Rock Mech Min Sci 34 (2) (1997) 199-205. 
[28] P. Perzyna, Fundamental problems in viscoplasticity, Adv Appl Mech 9 (1966) 243-377.

[29] Y. Jia, H. B. Bian, G. Duveau, K. Su, J. F. Shao, Hydromechanical mod770

[19] H. A. M. Van Eekelen, Isotropic yield surfaces in three dimensions for use in soil mechanics, Int J Numer Anal Meth Geomech 4 (1) (1980) 98-101.

[20] J. D. Barnichon, Finite Element Modelling in Structural and Petroleum Geology, Ph.D. thesis, Faculté des Sciences Appliquées, Université de Liège, Belgium (1998).

[21] L. Chen, J. F. Shao, H. W. Huang, Coupled elastoplastic damage modeling of anisotropic rocks, Comput Geotech 37 (1-2) (2010) 187-194.

[22] S. Pietruszczak, G. N. Pande, Description of soil anisotropy based on multilaminate framework, Int J Numer Anal Meth Geomech 25 (2) (2001) 197206.

[23] S. Pietruszczak, D. Lydzba, J. F. Shao, Modelling of inherent anisotropy in sedimentary rocks, Int J Solids Struct 39 (3) (2002) 637-648.

[24] S. Pietruszczak, Fundamentals of plasticity in geomechanics, CRC Press/Balkema, Leiden, The Netherlands, 2010, Ch. 7, Description of inherent anisotropy in geomaterials, pp. 133-156.

[25] S. Pietruszczak, Z. Mroz, Formulation of anisotropic failure criteria incorporating a microstructure tensor, Comput Geotech 26 (2000) 105-112.

[26] J. F. Shao, Q. Z. Zhu, K. Su, Modeling of creep in rock materials in terms of material degradation, Comput Geotech 30 (7) (2003) 549-555.

[27] J. F. Shao, K. T. Chau, X. T. Feng, Modeling of anisotropic damage and creep deformation in brittle rocks, Int J Rock Mech Min Sci 43 (4) (2006) $582-592$. elling of shaft excavation in Meuse/Haute-Marne laboratory, Phys Chem Earth 33 (2008) S422-S435. 
[30] H. Zhou, Y. Jia, J. F. Shao, A unified elastic-plastic and viscoplastic damage model for quasi-brittle rocks, Int J Rock Mech Min Sci 45 (8) (2008) $1237-1251$.

[31] R. D. Mindlin, Second gradient of strain and surface-tension in linear elasticity, Int J Solids Struct 1 (1965) 417-438.

[32] T. Matsushima, R. Chambon, D. Caillerie, Large strain finite element analysis of a local second gradient model: application to localization, Int $\mathrm{J}$ Numer Meth Engng 54 (4) (2002) 499-521.

[33] R. Chambon, D. Caillerie, N. E. Hassan, One-dimensional localisation studied with a second grade model, Eur J Mech A-Solid 17 (4) (1998) 637-656.

[34] F. Collin, D. Caillerie, R. Chambon, Analytical solutions for the thickwalled cylinder problem modeled with an isotropic elastic second gradient constitutive equation, Int J Solids Struct 46 (22-23) (2009) 3927-3937.

[35] M. T. van Genuchten, A closed-form equation for predicting the hydraulic conductivity of unsaturated soils, Soil Sci Soc Am J 44 (5) (1980) 892-898.

[36] R. Charlier, F. Collin, B. Pardoen, J. Talandier, J. P. Radu, P. Gerard, An unsaturated hydro-mechanical modelling of two in-situ experiments in Callovo-Oxfordian argillite, Eng Geol 165 (2013) 46-63.

[37] Andra, Dossier 2005 Référentiel du site Meuse/Haute-Marne tome 2 : Caractérisation comportementale du milieu géologique sous perturbation, Seconde édition, Paris, France (2005).

[38] R. Charlier, J. P. Radu, F. Collin, Projet HAVL - Argile - Expertise sur les mesures sur échantillons d'argilite du module de déformation et de la résistance à la compression simple, Tech. Rep. C.RP.0ULg.08.001, Université de Liège, Andra (2008).

[39] B. Pardoen, Hydro-mechanical analysis of the fracturing induced by the excavation of nuclear waste repository galleries using shear banding, Ph.D. 
thesis, Faculté des Sciences Appliquées, Université de Liège, Belgium (2015).

[40] P. Bésuelle, R. Chambon, F. Collin, Switching deformation modes in postlocalization solutions with a quasibrittle material, J Mech Mater Struct 1 (7) (2006) 1115-1134.

[41] M. Panet, A. Guenot, Analysis of convergence behind the face of a tunnel, in: Proceedings of the 3rd International Symposium : Tunnelling 82, Institution of Mining and Metallurgy, Brighton, 1982, pp. 197-204.

[42] B. Pardoen, J. Talandier, F. Collin, Permeability evolution and water transfer in the excavation damaged zone of a ventilated gallery, Int J Rock Mech Min Sci, under review.

[43] B. Pardoen, S. Levasseur, F. Collin, Excavation damaged zone modelling including hydraulic permeability evolution in unsaturated argilaceous rock, in: N. Khalili, A. R. Russell, A. Khoshghalb (Eds.), Unsaturated Soils:

【 Research and Applications, CRC Press, London, 2014, pp. 1387-1393. doi : 10.1201/b17034-203. 